\title{
A NONCOMMUTATIVE PROBABILITY THEORY
}

BY

\author{
S. P. GUDDER AND R. L. HUDSON
}

\begin{abstract}
A noncommutative probability theory is developed in which no boundedness, finiteness, or "tracial" conditions are imposed. The underlying structure of the theory is a "probability algebra" $(\mathbb{Q}, \omega)$ where $\mathbb{Q}$ is a *-algebra and $\omega$ is a faithful state on $\mathbb{Q}$. Conditional expectations and coarse-graining are discussed. The bounded and unbounded commutants are considered and commutation theorems are proved. Two classes of probability algebras, which we call closable and symmetric probability algebras are shown to have important regularity properties. The canonical algebra of quantum mechanics is considered in some detail and a strong commutation theorem is proven for this case. Moreover, in this case, isotropic normal states, KMS states, and stable states are defined and characterized.
\end{abstract}

1. Introduction. This paper develops a framework for a noncommutative probability theory which encompasses both the classical and quantum theories. Unlike previous work in this direction, we do not impose boundedness, finiteness, or "tracial" conditions [5], [8], [12], [16], [18]. This enables us to treat unbounded observables which, although occurring naturally in quantum mechanics, have been largely avoided in previous theories of this type. The paper falls into two main parts. The first part $(\$ \$ 1-6)$ develops the concepts and results of the abstract theory. The second part (\$\$7-9) illustrates some of these concepts and extends results of the first part for a specific example which is of particular interest in quantum mechanics.

A *algebra $\left(\mathbb{Q},{ }^{*}\right)$ is an associative, distributive, complex algebra $\mathbb{Q}$ with an identity 1 and an involution * satisfying

$$
x^{* *}=x, \quad(x y)^{*}=y^{*} x^{*}, \text { and }(\alpha x+y)^{*}=\alpha^{*} x^{*}+y^{*},
$$

for all $x, y \in \mathbb{Q}, \alpha \in \mathbf{C}$, where $\alpha^{*}$ denotes the complex conjugate of $\alpha \in \mathbf{C}$. A linear functional $\omega: \mathbb{Q} \rightarrow \mathbf{C}$ which is positive $\left(\omega\left(x^{*} x\right) \geqslant 0\right.$ for all $\left.x \in \mathbb{Q}\right)$ and normalized $(\omega(1)=1)$ is called a state. A state $\omega$ is faithful if $\omega\left(x^{*} x\right)=0$ implies $x=0$. The triple $\left(\mathscr{Q},{ }^{*}, \omega\right)$ where $\omega$ is a faithful state is called a probability algebra. We now illustrate, this concept with two examples.

Received by the editors January 12, 1977.

AMS (MOS) subject classifications (1970). Primary 46K10, 47C10; Secondary 46L10, 81A09, 81A17, 81A20.

Key words and phrases. Noncommutative probability, quantum probability, quantum mechanics, *algebras, unbounded representations, commutants. 
Let $(\Omega, \mathscr{F}, \mu)$ be a probability space and let $Q$ be the set of complex-valued, measurable simple functions. We identify functions that are equal almost everywhere so $\mathbb{Q}$ is actually composed of equivalence classes of simple functions. Under the pointwise operations of addition, multiplication, scalar multiplication, and complex conjugation ${ }^{*},\left(\mathscr{Q},{ }^{*}\right)$ becomes a ${ }^{*}$-algebra. Define $\omega: \mathbb{Q} \rightarrow \mathrm{C}$ by $\omega\left(\sum \alpha_{i} \chi_{A_{i}}\right)=\Sigma \alpha_{i} \mu\left(A_{i}\right)$. Then $\omega$ is a faithful state and $\left(\mathbb{Q},{ }^{*}, \omega\right)$ is a probability algebra. It is usually necessary to complete $\mathbb{Q}$ to a larger, more useful, class of functions. This is conveniently done by defining the inner product $\langle x, y\rangle=\omega\left(y^{*} x\right)$ and forming the Hilbert space completion $H=L^{2}(\Omega, \mathscr{F}, \mu)$ of $\mathcal{Q}$. Since $\omega$ is continuous on $\mathcal{Q}, \omega$ can be extended uniquely to a continuous linear functional on $H$.

In quantum mechanics one frequently has a complex algebra $\mathcal{Q}$ of operators on a common dense invariant domain $D$ of a Hilbert space $H$ which satisfies: $I \mid D \in \mathbb{Q}$ where $I$ is the identity operator; if $A^{\dagger}$ denotes the adjoint of $A$, then for all $A \in \mathbb{Q}, D \subseteq D\left(A^{\dagger}\right)$ and $A^{*}=A^{\dagger} \mid D \in \mathbb{Q}$. Then, under the usual operations, $\left(\mathbb{Q},{ }^{*}\right)$ is a ${ }^{*}$-algebra. Let $\psi \in D$ be a unit vector such that $A \psi=0$ implies $A=0$. If we define $\omega(A)=\langle A \psi, \psi\rangle$, then $\omega$ is a faithful state and $(Q, *, \omega)$ is a probability algebra.

2. Conditional expectation. Let $\left(\mathscr{Q},{ }^{*}, \omega\right)$ be a probability algebra and define the inner product $\langle x, y\rangle=\omega\left(y^{*} x\right)$. Notice that $\langle x y, z\rangle=\left\langle y, x^{*} z\right\rangle$ for all $x$, $y, z \in \mathbb{Q}$. Let $H$ be the Hilbert space completion of $\mathcal{Q}$. Since $\omega$ is continuous on $\mathbb{Q}$, it has a unique, continuous, linear extension to $H$ which we also denote by $\omega$. For $x \in \mathbb{Q}$, define the operator $\pi(x): \mathbb{Q} \rightarrow \mathbb{Q}$ by $\pi(x) y=x y$. Then $\pi$ is $a^{*}$-representation of $\mathcal{Q}$ on the dense invariant domain $D(\pi)=\mathbb{Q} \subseteq H$ [13]. The adjoint representation $\pi^{*}$ is defined as follows:

$$
D\left(\pi^{*}\right)=\cap\left\{D\left(\pi(x)^{*}\right): x \in \mathbb{Q}\right\}, \pi^{*}(x)=\left[\pi\left(x^{*}\right)\right]^{*} \mid D\left(\pi^{*}\right)
$$

[13]. Notice that $\mathbb{Q}=D(\pi) \subseteq D\left(\pi^{*}\right)$ and $\pi \subseteq \pi^{*}$.

Denote the set of operators $\{C: \mathbb{Q} \rightarrow H\}$ by $L(\mathbb{Q})$ and the set of bounded operators on $H$ by $L(H)$. An operator $C \in L(\mathbb{Q})$ is symmetric (nonnegative) if $\langle C x, y\rangle=\langle x, C y\rangle(\langle C x, x\rangle \geqslant 0)$ for all $x, y \in \mathbb{Q}$. Notice that a nonnegative operator is symmetric. Define the unbounded commutant $\pi(\mathbb{Q})^{c}$ of $\pi$ as

$$
\pi(\mathbb{Q})^{c}=\left\{C \in L(\mathbb{Q}):\langle C \pi(x) y, z\rangle=\left\langle C y, \pi\left(x^{*}\right) z\right\rangle, x, y, z \in \mathbb{Q}\right\} .
$$

The commutant $\pi(\mathscr{Q})^{\prime}$ of $\pi$ is

$$
\pi(\mathbb{Q})^{\prime}=\left\{C \in L(H): C \mid \mathbb{Q} \in \pi(\mathbb{Q})^{c}\right\} .
$$

If $C \in \pi(\mathrm{A})^{c}$, then $C: \mathbb{Q} \rightarrow D\left(\pi^{*}\right)$ and $C \pi(x)=\pi^{*}(x) C$ for all $x \in \mathbb{Q}$ [13].

A linear functional $F: \mathbb{Q} \rightarrow \mathbf{C}$ is absolutely continuous (denoted $F<\omega$ ) if $\omega\left(x_{i}^{*} x_{i}\right) \rightarrow 0$ implies $F\left(y x_{i}\right) \rightarrow 0$ for every $y \in \mathbb{Q}$. If $\nu$ is a state on $\left(\mathbb{Q},{ }^{*}, \omega\right)$ we say that $\nu$ is dominated (denoted $\nu \prec \omega$ ) if there exists an $M>0$ such that $\nu\left(x^{*} x\right) \leqslant M \omega\left(x^{*} x\right)$ for all $x \in \mathbb{Q}$. Applying Schwarz's inequality, 


$$
\left|\nu\left(y x_{i}\right)\right|<\nu\left(y y^{*}\right)^{1 / 2} \nu\left(x_{j}^{*} x_{j}\right)^{1 / 2},
$$

we see that $\nu \prec \omega$ implies $\nu<\omega$.

To illustrate these concepts, let $\left(\mathscr{Q},{ }^{*}, \omega\right)$ be the probability algebra of simple functions on $(\Omega, \mathcal{F}, \mu)$ considered in $\S 1$. If $\nu$ is a state on $\left(\mathbb{Q},{ }^{*}, \omega\right)$, then $\nu$ determines a measure $\nu_{0}(A)=\nu\left(\chi_{A}\right), A \in \mathscr{F}$. By the way $\left(\mathbb{Q},{ }^{*}, \omega\right)$ is defined, $\nu_{0}$ is automatically absolutely continuous relative to $\mu$ in the sense of measures. Now suppose $\nu<\omega$. Since $\nu$ is continuous on $H$, by the Riesz theorem, there exists an $f \in H$ such that $\nu(x)=\langle x, f\rangle=\int f x d \mu$. Hence $\nu_{0}(A)=\int_{A} f d \mu$ and $d \nu_{0} / d \mu=f \in H$. We thus see that $\nu<\omega$ if and only if $\nu_{0}$ is absolutely continuous relative to $\mu$ and $d \nu_{0} / d \mu \in H$. Suppose $\nu \prec \omega$. If $\mathcal{Q} \ni x_{i}>0$ and $x_{i} \rightarrow 0$ in $L^{1}(\Omega, \mathcal{F}, \mu)$, then

$$
\nu\left(x_{i}\right)=\nu\left(x_{i}^{1 / 2} x_{i}^{1 / 2}\right)<M \omega\left(x_{i}^{1 / 2} x_{i}^{1 / 2}\right)=M \int\left|x_{i}\right| d \mu \rightarrow 0 .
$$

In general, if $\mathcal{Q} \ni x_{i} \rightarrow 0$ in $L^{1}(\Omega, \mathscr{F}, \mu)$, then $x_{i}$ is a linear combination of four nonnegative functions all of which approach 0 in $L^{1}(\Omega, \mathcal{F}, \mu)$ so by linearity, $\nu\left(x_{i}\right) \rightarrow 0$. Hence $\nu$ can be extended to a linear functional in the dual $L^{\infty}(\Omega, \mathscr{F}, \mu)$ of $L^{1}(\Omega, \mathscr{F}, \mu)$. Thus there is an essentially bounded function $f$ such that $\nu(x)=\int f x d \mu$. We then see that $\nu \prec \omega$ if and only if $\nu_{0}$ is absolutely continuous relative to $\mu$ and $d \nu_{0} / d \mu$ is essentially bounded.

THEOREM 1. (a) A linear functional $F$ on $\left(\mathscr{Q},{ }^{*}, \omega\right)$ is absolutely continuous if and only if there exists a $C \in \pi(\mathbb{Q})^{c}$ such that $F(x)=\left[\omega\left(C x^{*}\right)\right]^{*}$ for all $x \in \mathbb{Q}, C$ is unique and is denoted $C=d F / d \omega$.

(2) A functional $\nu$ on $\left(\mathscr{Q},{ }^{*}, \omega\right)$ is an absolutely continuous state if and only if there exists a nonnegative $C \in \pi(\mathbb{Q})^{c}$ such that $\omega(C 1)=1$ and $\nu(x)=\omega(C x)$ for all $x \in \mathbb{Q}, C$ is strictly positive if and only if $\nu$ is faithful. The following statements are equivalent: (a) $C$ is bounded; (b) $\nu \prec \omega$; (c) $\omega\left(x_{i}^{*} x_{i}\right) \rightarrow 0$ implies $\nu\left(x_{i}^{*} x_{i}\right) \rightarrow 0$.

Proof. (1) Suppose $F<\omega$. Then for any $y \in \mathbb{Q}, x \rightarrow F\left(y^{*} x\right)$ is a continuous linear functional on $\mathbb{Q}$. By the Riesz theorem, there exists a $y^{\prime} \in H$ such that $F\left(y^{*} x\right)=\left\langle x, y^{\prime}\right\rangle$ for every $x \in \mathbb{Q}$. Define $C: \mathbb{Q} \rightarrow H$ by $C y=y^{\prime}$. It is straightforward to show that $C$ is linear. To show $C \in \pi(\mathbb{Q})^{c}$, for every $x, y, z \in \mathbb{Q}$ we have

$$
\begin{aligned}
\langle C \pi(x) y, z\rangle & =\langle z, C x y\rangle^{*}=\left[F\left(y^{*} x^{*} z\right)\right]^{*} \\
& =\left\langle x^{*} z, C y\right\rangle^{*}=\left\langle C y, \pi\left(x^{*}\right) z\right\rangle .
\end{aligned}
$$

Furthermore,

$$
\begin{aligned}
F(x) & =\langle x, C 1\rangle=\langle\pi(x) 1, C 1\rangle=\left\langle 1, C x^{*}\right\rangle \\
& =\left\langle C x^{*}, 1\right\rangle^{*}=\left[\omega\left(C x^{*}\right)\right]^{*} .
\end{aligned}
$$

Conversely, suppose $F(x)=\omega\left(C x^{*}\right)^{*}$ for $C \in \pi(\mathbb{Q})^{c}$. Then 


$$
|F(y x)|=\left|\omega\left(C x^{*} y^{*}\right)\right|=\left|\left\langle C x^{*} y^{*}, 1\right\rangle\right|=\left|\left\langle C y^{*}, x\right\rangle\right|\left\langle\left\|C y^{*}\right\|\|x\|\right.
$$

so $F<\omega$. To show $C$ is unique, suppose $F(x)=\omega\left(C_{1} x^{*}\right)^{*}$ for $C_{1} \in \pi(\mathbb{Q})^{c}$. Then for $x, y \in \mathbb{Q}$ we have

$$
\begin{aligned}
\left\langle C_{1} y, x\right\rangle & =\left\langle C_{1} x^{*} y, 1\right\rangle=\omega\left(C_{1} x^{*} y\right)=\left[F\left(y^{*} x\right)\right]^{*} \\
& =\omega\left(C x^{*} y\right)=\langle C y, x\rangle .
\end{aligned}
$$

(2) Suppose $\nu$ is a state and $\nu<\omega$. Then by (1) there exists a $C \in \pi(\mathbb{Q})^{c}$ such that $\nu\left(y^{*} x\right)=\langle x, C y\rangle$ for every $x, y \in \mathbb{Q}$. Now $C$ is nonnegative since $\langle x, C x\rangle=\nu\left(x^{*} x\right) \geqslant 0$ and $\omega(C 1)=\langle C 1,1\rangle=\nu(1)=1$. Also, $\nu(x)=$ $\langle x, C 1\rangle=\langle C x, 1\rangle=\omega(C x)$. Conversely, if $\nu(x)=\omega(C x)$ where $C \in \pi(\mathbb{Q})^{c}$ is nonnegative and $\omega(C 1)=1$, then $\nu$ is a state since

$$
\nu\left(x^{*} x\right)=\omega\left(C x^{*} x\right)=\left\langle C x^{*} x, 1\right\rangle=\langle C x, x\rangle>0 .
$$

That $\nu<\omega$ follows from (1). Since $\nu\left(x^{*} x\right)=\langle C x, x\rangle$ we see that $\nu$ is faithful if and only if $C$ is strictly positive. (a) $\Rightarrow$ (b) If $C$ is bounded, then

$$
\nu\left(x^{*} x\right)=\langle C x, x\rangle\left\langle\|C\|\|x\|^{2}=\|C\| \omega\left(x^{*} x\right)\right. \text {. }
$$

(b) $\Rightarrow$ (c) trivial. (c) $\Rightarrow$ (a) Suppose $\omega\left(x_{i}^{*} x_{i}\right) \rightarrow 0$ implies $\nu\left(x_{i}^{*} x_{i}\right) \rightarrow 0$. Since $C$ is nonnegative and densely defined, $C$ has a selfadjoint extension and, hence, $C$ has a nonnegative square root $T$. If $\mathbb{Q} \ni x_{i} \rightarrow 0$ then $\omega\left(x_{i}^{*} x_{i}\right) \rightarrow 0$ so

$$
\left\langle T x_{i}, T x_{i}\right\rangle=\left\langle T^{2} x_{i}, x_{i}\right\rangle=\left\langle C x_{i}, x_{i}\right\rangle=\nu\left(x_{i}^{*} x_{i}\right) \rightarrow 0 .
$$

Hence $T x_{i} \rightarrow 0$ and $T$ is bounded on $Q$. Therefore, $C=T^{2}$ is bounded.

Corollary. $\nu \prec \omega$ if and only if there exists a nonnegative $C \in \pi(\mathbb{Q})^{\prime}$ satisfying $\omega(C 1)=1$ and $\nu(x)=\omega(C x)$ for all $x \in \mathbb{Q}$.

COROLlary. If $\nu<\omega$ then there exists a unique $\hat{z} \in D\left(\pi^{*}\right)$ such that $\nu(x)=\omega\left[\pi^{*}(x) \hat{z}\right]$ for all $x \in \mathbb{Q}$.

Proof. $\nu(x)=\omega(C x)=\omega[C \pi(x) 1]=\omega\left[\pi^{*}(x) C 1\right]$.

Corollary. If $\nu, \eta<\omega$ and $(d v / d \omega) 1=(d \eta / d \omega) 1$ then $\nu=\eta$.

Let $\left(\mathbb{Q},{ }^{*}, \omega\right)$ be a probability algebra and let $\mathscr{B} \subseteq \mathbb{Q}$ be a ${ }^{*}$-subalgebra. Then $\mathscr{B}$ is a subspace of $H$ and its closure is a closed subspace $\overline{\mathscr{B}}$. Let $\boldsymbol{P}_{\mathscr{B}}$ denote the orthogonal projection onto $\overline{\mathscr{B}}$. We denote the restriction of $\pi$ to $\mathscr{B}$ with domain the dense subspace $\mathscr{B}$ of $\overline{\mathscr{B}}$ by $\pi \mid \mathfrak{B}$. Thus $\pi \mid \mathscr{B}$ is a *-representation of $\mathscr{B}$ with dense invariant domain $D(\pi \mid \mathscr{B})=\mathscr{B} \subseteq \overline{\mathscr{B}}$. For $x \in \mathbb{Q}$, the conditional expectation $E(x \mid \mathscr{B})$ of $x$ given $\mathscr{B}$ is defined by

$$
\begin{gathered}
E(x \mid \mathfrak{B}) \in D\left[(\pi \mid \mathfrak{B})^{*}\right] \subseteq \overline{\mathscr{B}}, \\
\omega(y x)=\omega\left[(\pi \mid \mathfrak{B})^{*}(y) E(x \mid \mathfrak{B})\right] \text { for all } y \in \mathscr{B} .
\end{gathered}
$$

THEOREM 2. (1) $E(x \mid \Re)$ exists, is unique and $E(x \mid \Re)=P_{\mathscr{B}} x$.

(2) A function $F: \mathbb{Q} \rightarrow D\left[(\pi \mid \mathscr{B})^{*}\right]$ equals $E(\cdot \mid \mathscr{B})$ if and only if $F$ satisfies 
$\omega[F(x)]=\omega(x)$ and $F(y x)=(\pi \mid \mathscr{B})^{*}(y) F(x)$ for all $x \in \mathbb{Q}, y \in \mathscr{B}$.

(3) If for all $x \in \mathbb{Q}$, we define $\omega_{x}: \mathscr{B} \rightarrow \mathbf{C}$ by $\omega_{x}(y)=\omega(x y)$, then $\omega_{x}<\omega \mid \mathscr{B}$ and $E(x \mid \Re)=\left(d \omega_{x^{*}} / d \omega \mid \Re\right) 1$.

Proof. (1) To prove uniqueness, suppose $E$ and $E_{1}$ are conditional expectations. Then for every $x \in \mathbb{Q}, y \in \mathscr{B}$ we have

$$
\begin{gathered}
\langle E(x \mid \mathfrak{B}), y\rangle=\langle E(x \mid \mathfrak{B}),(\pi \mid \mathfrak{B})(y) 1\rangle=\left\langle(\pi \mid \mathfrak{B})(y)^{*} E(x \mid \mathfrak{B}), 1\right\rangle \\
=\left\langle(\pi \mid \mathfrak{B})^{*}\left(y^{*}\right) E(x \mid \mathfrak{B}), 1\right\rangle=\omega\left[(\pi \mid \mathfrak{B})^{*}\left(y^{*}\right) E(x \mid \mathfrak{B})\right] \\
=\omega\left(y^{*} x\right)=\omega\left[(\pi \mid \mathfrak{B})^{*}\left(y^{*}\right) E_{1}(x \mid \mathfrak{B})\right]=\left\langle E_{1}(x \mid \mathfrak{B}), y\right\rangle .
\end{gathered}
$$

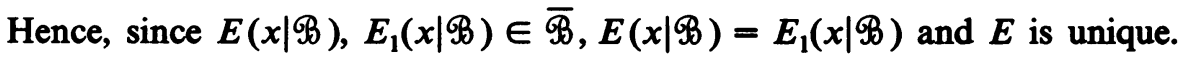
We now show that $P_{\mathscr{B}}$ is a conditional expectation. For every $x \in \mathbb{Q}, y$, $z \in \mathscr{B}$ we have

$$
\left|\left\langle\pi(z) y, P_{\mathscr{D}} x\right\rangle\right|=|\langle z y, x\rangle|=\left|\left\langle y, z^{*} x\right\rangle\right|\left\langle\left\|z^{*} x\right\|\|y\| .\right.
$$

Hence $P_{\mathscr{B}} x \in D\left(\pi(z)^{*}\right)$ for all $z$ so $P_{\mathscr{B}} x \in D\left[(\pi \mid \mathscr{B})^{*}\right]$. To show that $P_{\mathscr{B}} x$ satisfies (2.2), for all $y \in \mathscr{B}$ we have

$$
\omega\left[(\pi \mid \mathscr{B})^{*}(y) P_{\mathscr{B}} x\right]=\left\langle P_{\mathscr{B}} x, y^{*}\right\rangle=\left\langle x, y^{*}\right\rangle=\omega(y x) .
$$

(2) Letting $y=1$ in (2.2) gives $\omega[E(x \mid \mathscr{B})]=\omega(x)$. Also, for all $x \in \mathbb{Q}, y$, $z \in \mathscr{B}$ we have

$$
\begin{aligned}
\langle E(y x \mid \mathfrak{B}), z\rangle & =\omega\left[(\pi \mid \mathfrak{B})^{*}(z) E(y x \mid \mathfrak{B})\right]=\omega(z y x) \\
& =\omega\left[(\pi \mid \mathfrak{B})^{*}(z y) E(x \mid \mathfrak{B})\right]=\omega\left[(\pi \mid \mathfrak{B})^{*}(z)(\pi \mid \mathfrak{B})^{*}(y) E(x \mid \mathfrak{B})\right] \\
& =\left\langle(\pi \mid \mathfrak{B})^{*}(y) E(x \mid \mathfrak{B}), z\right\rangle .
\end{aligned}
$$

Hence $E(y x \mid \mathscr{B})=(\pi \mid \mathscr{B})^{*}(y) E(x \mid \mathscr{B})$. Conversely, if $F$ satisfies the requirements of (2) then for all $x \in \mathbb{Q}, y \in \mathscr{B}$ we have

$$
\omega\left[(\pi \mid \mathfrak{B})^{*}(y) F(x)\right]=\omega[F(y x)]=\omega(y x) .
$$

By uniqueness, $F=E(\cdot \mid \Re)$.

(3) To show $\omega_{x}<\omega \mid \mathscr{B}$, suppose $x_{i} \in \mathscr{B}$ and $\omega\left(x_{i}^{*} x_{i}\right) \rightarrow 0$. If $y \in \mathscr{B}$ we have

$$
\left|\omega_{x}\left(y x_{i}\right)\right|=\left|\omega\left(x y x_{i}\right)\right|<\omega\left(x y y^{*} x\right)^{1 / 2} \omega\left(x_{i}^{*} x_{i}\right)^{1 / 2} \rightarrow 0 .
$$

Hence

$$
d \omega_{x^{*}} / d \omega \mid \mathscr{B} \in(\pi \mid \mathfrak{B})(\mathscr{B})^{c} \text { and }\left(d \omega_{x^{*}} / d \omega \mid \mathscr{B}\right) 1 \in D\left[(\pi \mid \mathfrak{B})^{*}\right] .
$$

Furthermore, for all $y \in \mathscr{B}$ we have

$$
\begin{aligned}
& \omega\left[(\pi \mid \mathscr{B})^{*}(y)\left(d \omega_{x^{*}} / d \omega \mid \mathscr{B}\right) 1\right]=\omega\left[\left(d \omega_{x^{*}} / d \omega \mid \mathscr{B}\right)(\pi \mid \mathscr{B})(y) 1\right] \\
& =\omega\left[\left(d \omega_{x^{*}} / d x \mid \mathscr{B}\right) y\right]=\left[\omega_{x^{*}}\left(y^{*}\right)\right]^{*}=\left[\omega\left(x^{*} y^{*}\right)\right]^{*}=\omega(y x) .
\end{aligned}
$$


The result now follows from uniqueness.

3. Coarse-graining. Let the ${ }^{*}$-algebra $\left(Q,{ }^{*}\right)$ represent some physical or statistical system. Suppose we have an a priori distribution given by a faithful state $\omega$. Then $\left(\mathscr{Q},{ }^{*}, \omega\right)$ is a probability algebra. We can now predict the behaviour of the system in terms of $\omega$, but suppose we want to make a more precise prediction. In practice, it is impossible to measure all the observables in $\mathcal{Q}$, so we make a partial measurement. That is, we measure observables in a *-subalgebra $\mathscr{B} \subseteq \mathbb{Q}$. This gives a state $\nu$ on $\mathscr{B}$. We would now like to extend $\nu$ to a state $\nu_{c}$ on $\mathscr{Q}$ which gives no additional information. If this is possible, we call $\nu_{c}$ a coarse-graining of $\nu$. This procedure is frequently used in quantum mechanics and in statistical inference [8], [10].

More precisely, let $\left(\mathscr{Q},{ }^{*}, \omega\right)$ be a probability algebra and let $\mathscr{B} \subseteq \mathbb{Q}$ be a *-subalgebra. Let $\nu$ be a state on $\mathscr{B}$ such that $\nu<\omega \mid \mathscr{B}$. A $(\mathscr{B}, \omega)$ coarsegraining of $\nu$ is a state $\nu_{c}$ on $\mathscr{Q}$ satisfying:

(3.1) $\nu_{\mathrm{c}} \mid \Re^{\prime}=\nu$,

(3.2) $\nu_{c}<\omega$,

(3.3) $\nu_{c}(x)=\nu_{c}[E(x \mid \mathfrak{B})]$ for all $x \in \mathbb{Q}$.

$\mathrm{A}^{*}$-subalgebra $\mathscr{B} \subseteq \mathbb{Q}$ is positivity preserving if for any $x \in \mathbb{Q}$ there exists a sequence $y_{i} \in \mathscr{B}$ such that $y_{i}^{*} y_{i} \rightarrow P_{\mathscr{B}} x^{*} x$. In the case of the probability algebra $(Q, *, \omega)$ of measurable simple funcitons on $(\Omega, \mathscr{F}, \mu)$, any ${ }^{*}$-subalgebra $\mathscr{B} \subseteq \mathbb{Q}$ is positivity preserving.

Theorem 3. Let $\mathscr{B} \subseteq \mathbb{Q}$ be $a^{*}$-subalgebra and let $\nu$ be a state on $\mathscr{B}$ such that $\nu<\omega \mid \mathfrak{B}$.

(1) The following statements are equivalent:

(a) $A(\Re, \omega)$ coarse-graining of $\nu$ exists; $\bar{B}$;

(b) $\nu$ has an absolutely continuous extension $\nu_{1}$ to $\mathbb{Q}$ such that $\left(d \nu_{1} / d \omega\right) 1 \in$

(c) $d \nu / d \omega \mid \Re$ has a nonnegative extension in $\pi(\mathscr{Q})^{c}$.

(2) If $a(\mathscr{B}, \omega)$ coarse-graining $\nu_{c}$ of $\nu$ exists it is unique and satisfies

(d) $\nu_{c}(x)=\nu[E(x \mid \Re)]$ for all $x \in \mathbb{Q}$;

(e) $\nu_{c}$ is the unique absolutely continuous extension of $\nu$ to $\mathbb{Q}$ such that $\left(d v_{c} / d \omega\right) 1 \in \overline{\mathscr{B}}$;

(f) $\nu_{c}$ is the unique absolutely continuous extension of $\nu$ to $\mathbb{Q}$ such that $\left(d \nu_{c} / d \omega\right)|\Re=d \nu / d \omega| \Re$.

(3) If $\nu \prec \omega \mid \mathscr{B}$ and $\mathscr{B}$ is positivity preserving, then $\nu_{c}$ exists and $\nu_{c} \prec \omega$.

Proof. (1) (a) $\Rightarrow$ (b) Suppose a $(\mathscr{B}, \omega)$ coarse-graining $\nu_{c}$ of $\nu$ exists. Then $\nu_{c}$ is an absolutely continuous extension of $\nu$ to $\mathbb{Q}$. If $x \in \mathbb{Q}$ and $\mathscr{B} \ni y_{i} \rightarrow$ $P_{\mathscr{P}} x$ we have 


$$
\begin{aligned}
\left\langle x,\left(d \nu_{c} / d \omega\right) 1\right\rangle & =\left\langle\left(d \nu_{c} / d \omega\right) x, 1\right\rangle=\omega\left[\left(d \nu_{c} / d \omega\right) x\right] \\
& =\nu_{c}(x)=\nu_{c}\left(P_{\mathscr{B}} x\right)=\lim \nu_{c}\left(y_{i}\right)=\lim \omega\left[\left(d \nu_{c} / d \omega\right) y_{i}\right] \\
& =\lim \left\langle\left(d \nu_{c} / d \omega\right) y_{i}, 1\right\rangle=\lim \left\langle y_{i},\left(d \nu_{c} / d \omega\right) 1\right\rangle \\
& =\left\langle P_{\mathscr{B}} x,\left(d \nu_{c} / d \omega\right) 1\right\rangle=\left\langle x, P_{\mathscr{B}}\left(d \nu_{c} / d \omega\right) 1\right\rangle .
\end{aligned}
$$

Hence $\left(d \nu_{c} / d \omega\right) 1=P_{\mathscr{P}}\left(d \nu_{c} / d \omega\right) 1 \in \overline{\mathscr{B}}$.

(b) $\Rightarrow$ (c) Suppose $\nu$ has an absolutely continuous extension $\nu_{1}$ to $\mathbb{Q}$ such that $\left(d \nu_{1} / d \omega\right) 1 \in \overline{\mathscr{B}}^{\mathrm{B}}$. Then $d \nu_{1} / d \omega \in \pi(\mathbb{Q})^{c}$, and for any $y \in \mathscr{B}$ we have

$$
\left\langle y,\left(d \nu_{1} / d \omega\right) 1\right\rangle=\nu_{1}(y)=\nu(y)=\nu(y)=\langle y,(d \nu / d \omega \mid \mathscr{B}) 1\rangle .
$$

Hence, $\left(d \nu_{1} / d \omega\right) 1=(d \nu / d \omega \mid \mathscr{B}) 1$ and for any $y \in \mathscr{B}$ we have

$$
\begin{aligned}
(d \nu / d \omega \mid \Re) y & =(d \nu / d \omega \mid \Re) \pi(y) 1=\pi^{*}(y)(d \nu / d \omega \mid \Re) 1 \\
& =\pi^{*}(y)\left(d \nu_{1} / d \omega\right) 1=\left(d \nu_{1} / d \omega\right) y .
\end{aligned}
$$

Thus $d \nu_{1} / d \omega$ is a nonnegative extension of $d \nu / d \omega \mid \mathfrak{B}$ in $\pi(\mathscr{Q})^{c}$.

(c) $\Rightarrow$ (a) Suppose $d \nu / d \omega \mid \mathscr{B}$ has a nonnegative extension $C \in \pi(\mathbb{Q})^{c}$. We now show that $\nu_{c}(x)=\nu[E(x \mid \mathscr{B})]$ is a $(\mathscr{B}, \omega)$ coarse-graining of $\nu$. Clearly, $\nu_{c}$ is linear on $\mathcal{Q}, \nu_{c}(1)=1$, and $\nu_{c}$ satisfies (3.1) and (3.3). If $x, y \in \mathbb{Q}$ and $\mathscr{B} \ni z_{i} \rightarrow P_{\mathscr{B}} y x$ we have

$$
\begin{aligned}
\nu_{c}(y x) & =\nu[E(y x \mid \mathfrak{B})]=\nu\left(P_{\mathscr{B}} y x\right)=\lim \nu\left(z_{i}\right) \\
& =\lim \omega\left[(d \nu / d \omega \mid \mathscr{B}) z_{i}\right]=\lim \left\langle(d \nu / d \omega \mid \mathscr{B}) z_{i}, 1\right\rangle \\
& =\lim \left\langle z_{i},(d \nu / d \omega \mid \mathscr{B}) 1\right\rangle=\left\langle P_{\mathscr{B}} y x,(d \nu / d \omega \mid \mathscr{B}) 1\right\rangle \\
& =\langle y x,(d \nu / d \omega \mid \mathfrak{B}) 1\rangle=\langle\pi(y) x, C 1\rangle=\left\langle x, C y^{*}\right\rangle .
\end{aligned}
$$

Hence $\nu_{c}\left(x^{*} x\right)=\langle x, C x\rangle \geqslant 0$ so $\nu_{c}$ is a state and $\left|\nu_{c}(y x)\right|<\left\|C y^{*}\right\|\|x\|$ so $\nu_{c}<\omega$ and (3.2) is satisfied.

(2) (d) Suppose a $(\Re, \omega)$ coarse-graining $\nu_{c}$ of $\nu$ exists. For $x \in \mathbb{Q}$, let $\mathscr{B} \ni y_{i} \rightarrow E(x \mid \mathscr{B})$. Then we have

$$
\nu_{c}(x)=\nu_{c}[E(x \mid \mathfrak{B})]=\lim \nu_{c}\left(y_{i}\right)=\lim \nu\left(y_{i}\right)=\nu[E(x \mid \mathfrak{B})] .
$$

(e) That $\left(d \nu_{c} / d \omega\right) 1 \in \overline{\mathscr{B}}$ follows from (a) $\Rightarrow$ (b) above. If $\nu_{1}$ is an absolutely continuous extension of $\nu$ to $\mathbb{Q}$ such that $\left(d \nu_{1} / d \omega\right) 1 \in \mathscr{\mathscr { B }}_{B}$, then from the proof of $(\mathrm{b}) \Rightarrow$ (c) above we have $\left(d \nu_{1} / d \omega\right) 1=\left(d \nu_{c} / d \omega\right) 1$. It follows from the third corollary of Theorem 1 that $\nu_{1}=\nu_{c}$.

(f) This follows from the proof of (b) $\Rightarrow$ (c) and (e) above.

(3) Suppose $\nu \prec \omega \mid \mathscr{B}$ and $\mathscr{B}$ is positivity preserving. We will show that $\nu_{c}(x)=\nu[E(x \mid \mathscr{B})]$ is a $(\mathscr{B}, \omega)$ coarse-graining of $\nu$ and that $\nu_{c} \prec \omega$. To show that $\nu_{c}$ is positive, let $x \in \mathbb{Q}$ and let $y_{i} \in \mathscr{B}$ be a sequence such that $y_{i}^{*} y_{i} \rightarrow P_{\mathscr{B}} x^{*} x$. Then

$$
\nu_{c}\left(x^{*} x\right)=\nu\left(P_{\mathscr{B}} x^{*} x\right)=\lim \nu\left(y_{i}^{*} y_{i}\right)>0 .
$$

To show that $\nu_{c} \prec \omega$, we have 


$$
\begin{aligned}
\nu_{c}\left(x^{*} x\right) & =\lim \nu\left(y_{i}^{*} y_{i}\right)=\lim \omega\left[(d \nu / d \omega \mid \mathscr{B}) y_{i}^{*} y_{i}\right] \\
& =\lim \left\langle(d \nu / d \omega \mid \mathscr{B}) y_{i}, y_{i}\right\rangle\left\langle\|d \nu / d \omega|\Re|\| \lim \left\langle y_{i}, y_{i}\right\rangle\right. \\
& =\left\|d \nu / d \omega\left|\mathscr{B}\left\|\left\langle P_{\mathscr{B}} x^{*} x, 1\right\rangle=\right\| d \nu / d \omega\right| \Re\right\| \omega\left(x^{*} x\right) .
\end{aligned}
$$

We now show that in a certain sense the $(\Re, \omega)$ coarse-graining of $\nu$ is the absolutely continuous extension of $\nu$ with minimal information. Let $(Q, *, \omega)$ be a probability algebra and suppose $\nu<\omega$ is a positive linear functional. We would like to define the information measure $I_{\omega}(\nu)$ of $\nu$ relative to $\omega$. Guided by the properties of a measure we shall postulate that $I_{\omega}$ be nonnegative and additive. Nonnegativity is simply $I_{\omega}(\nu) \geqslant 0$. To motivate additivity, consider the classical situation in which $\nu_{1}, \nu_{2}$ are mutually singular measures on a probability space $(\Omega, \mathscr{F}, \mu)$ which are absolutely continuous relative to $\mu$. Since $\nu_{1}, \nu_{2}$ are mutually singular there exist disjoint measurable sets $A_{1}, A_{2}$ such that $A_{1} \cup A_{2}=\Omega$ and $\nu_{1}\left(A_{2}\right)=\nu_{2}\left(A_{1}\right)=0$. It now seems reasonable to assume that $I_{\omega}\left(\nu_{1}+\nu_{2}\right)=I_{\omega}\left(\nu_{1}\right)+I_{\omega}\left(\nu_{2}\right)$. Notice that $\nu_{1}, \nu_{2}$ are mutually singular if and only if $\int\left(d \nu_{1} / d \mu\right)\left(d \nu_{2} / d \mu\right) d \mu=0$.

This reasoning motivates the following definition of information. If $F<\omega$ is a linear functional, the information $I_{\omega}(F)$ of $F$ relative to $\omega$ satisfies:

(3.4) $I_{\omega}(F) \geqslant 0, I_{\omega}(\omega)=1$;

(3.5) for $F_{1}, F_{2}<\omega$ with $\left\langle\left(d F_{1} / d \omega\right) 1,\left(d F_{2} / d \omega\right) 1\right\rangle=0, I_{\omega}\left(F_{1}+F_{2}\right)=$ $I_{\omega}\left(F_{1}\right)+I_{\omega}\left(F_{2}\right)$.

THEOREM 4. (1) $I_{\omega}(F)$ exists, is unique and $I_{\omega}(F)=\|(d F / d \omega) 1\|^{2}=$ $F[(d F / d \omega) 1]$. (2) If the $(\mathscr{B}, \omega)$ coarse-graining of $\nu$ exists, it is the unique absolutely continuous extension of $\nu$ to $\mathbb{Q}$ with minimal information relative to $\omega$.

Proof. (1) It is clear that $F \rightarrow\|(d F / d \omega) 1\|^{2}$ satisfies (3.4) and (3.5). To prove uniqueness, suppose $I_{\omega}$ satisfies (3.4) and (3.5). For $y \in D\left(\pi^{*}\right)$ define the linear functional $F_{y}: \mathbb{Q} \rightarrow \mathbf{C}$ by $F_{y}(x)=\langle x, y\rangle$. Now $F_{y}<\omega$ since for all $x, z \in \mathbb{Q}$ we have

$$
\left|F_{y}(z x)\right|=|\langle z x, y\rangle|=\left|\left\langle x, \pi(z)^{*} y\right\rangle\right|\left\langle\left\|\pi(z)^{*} y\right\|\|x\| .\right.
$$

Define the map $G: D\left(\pi^{*}\right) \rightarrow \mathbf{R}^{+}$by $G(y)=I_{\omega}\left(F_{y}\right)$. Notice that $\left(d F_{y} / d \omega\right) 1=$ $y$ since for all $x \in \mathbb{Q}$ we have

$$
\begin{aligned}
\left\langle\left(d F_{y} / d \omega\right) 1, x\right\rangle & =\left\langle\left(d F_{y} / d \omega\right) x^{*}, 1\right\rangle=\omega\left[\left(d F_{y} / d \omega\right) x^{*}\right] \\
& =\left[F_{y}(x)\right]^{*}=\langle x, y\rangle^{*}=\langle y, x\rangle .
\end{aligned}
$$

Let $y, z \in D\left(\pi^{*}\right)$ satisfy $\langle y, z\rangle=0$. Then $\left\langle\left(d F_{y} / d \omega\right) 1,\left(d F_{z} / d \omega\right) 1\right\rangle=0$ so

$$
\begin{aligned}
G(y+z) & =I_{\omega}\left(F_{y+z}\right)=I_{\omega}\left(F_{y}+F_{z}\right) \\
& =I_{\omega}\left(F_{y}\right)+I_{\omega}\left(F_{z}\right) \\
& =G(y)+G(z) .
\end{aligned}
$$

Applying a result of [9] there exists a $c>0$ such that $G(y)=c\|y\|^{2}$. 
If $F<\omega$, then $F=F_{(d F / d \omega) 1}$, since for all $x \in \mathbb{Q}$ we have

$$
\begin{aligned}
F(x) & =\left[\omega\left((d F / d \omega) x^{*}\right)\right]^{*}=\left\langle(d F / d \omega) x^{*}, 1\right\rangle^{*} \\
& =\langle(d F / d \omega) 1, x\rangle^{*}=\langle x,(d F / d \omega) 1\rangle .
\end{aligned}
$$

Hence

$$
I_{\omega}(F)=I_{\omega}\left[F_{(d F / d \omega) 1}\right]=G[(d F / d \omega) 1]=c\|(d F / d \omega) 1\|^{2} .
$$

Also, $1=I_{\omega}(\omega)=c\|(d \omega / d \omega) 1\|^{2}=c$. Finally, if $F<\omega$ and $\mathbb{Q} \ni x_{i} \rightarrow$ $(d F / d \omega) 1$ we have

$$
\begin{aligned}
I_{\omega}(F) & =\langle(d F / d \omega) 1,(d F / d \omega) 1\rangle=\lim \left\langle(d F / d \omega) 1, x_{i}\right\rangle \\
& =\lim \left\langle(d F / d \omega) x_{i}^{*}, 1\right\rangle=\lim \omega\left[(d F / d \omega) x_{i}^{*}\right] \\
& =\lim \left[F\left(x_{i}\right)\right]^{*}=F[(d F / d \omega) 1]^{*}=F[(d F / d \omega) 1] .
\end{aligned}
$$

(2) Suppose the $(\mathscr{B}, \omega)$ coarse-graining $\nu_{c}$ of $\nu$ exists. Suppose $F<\omega$ and $F \mid \mathscr{B}=\nu$. Since $\overline{\mathscr{B}}$ is a closed subspace of $H$, we have $(d F / d \omega) 1=x+y$ where $x \in \overline{\mathscr{B}}$ and $y \in \overline{\mathscr{B}}^{\perp}$. For $z \in \mathscr{B}$, since $d \nu / d \omega\left|\mathscr{B}=\left(d \nu_{c} / d \omega\right)\right| \mathscr{B}$ we have

$$
\begin{aligned}
\langle x, z\rangle & =\langle x+y, z\rangle=\langle(d F / d \omega) 1, z\rangle=\left\langle(d F / d \omega) z^{*}, 1\right\rangle \\
& =\omega\left[(d F / d \omega) z^{*}\right]=[F(z)]^{*}=\nu\left(z^{*}\right)=\omega\left[(d \nu / d \omega \mid \mathfrak{B}) z^{*}\right] \\
& =\omega\left[\left(d \nu_{c} / d \omega\right) z^{*}\right]=\left\langle\left(d \nu_{c} / d \omega\right) z^{*}, 1\right\rangle=\left\langle\left(d \nu_{c} / d \omega\right) 1, z\right\rangle .
\end{aligned}
$$

By Theorem 3(e), $\left(d \nu_{c} / d \omega\right) 1 \in \mathscr{B}$ so $x=\left(d \nu_{c} / d \omega\right) 1$. Hence

$$
\begin{aligned}
I_{\omega}(F) & =\|(d F / d \omega) 1\|^{2}=\|x\|^{2}+\|y\|^{2} \geqslant\|x\|^{2} \\
& =\left\|\left(d \nu_{c} / d \omega\right) 1\right\|^{2}=I_{\omega}\left(\nu_{c}\right) .
\end{aligned}
$$

Hence $I_{\omega}\left(\nu_{c}\right)$ is a minimum and $F$ has minimal information relative to $\omega$ if and only if $\|y\|=0$ in which case $(d F / d \omega) 1=\left(d \nu_{c} / d \omega\right) 1$ and by the third corollary of Theorem $1, F=\nu_{c}$.

Let $\left(\mathbb{Q}_{1},{ }^{*}\right)$ and $\left(\mathbb{Q}_{2},{ }^{*}\right)$ be ${ }^{*}$-algebras. Let $\mathbb{Q}_{1} \otimes \mathbb{Q}_{2}$ be the algebraic tensor product of $\mathbb{Q}_{1}$ and $Q_{2}$ with product defined by $\left(x_{1} \otimes x_{2}\right)\left(y_{1} \otimes y_{2}\right)=x_{1} y_{1} \otimes$ $x_{2} y_{2}$. If we define $\left(x_{1} \otimes x_{2}\right)^{*}=x_{1}^{*} \otimes x_{2}^{*}$, then $\left(Q_{1} \otimes \mathbb{Q}_{2},{ }^{*}\right)$ is a ${ }^{*}$-algebra. We call $\left(Q_{1},{ }^{*}\right),\left(\mathscr{Q}_{2},{ }^{*}\right)$ the component systems of the compound system $\left(Q_{1} \otimes \mathbb{Q}_{2},{ }^{*}\right)$. If $\left(\mathbb{Q}_{1},{ }^{*}\right),\left(\mathbb{Q}_{2},{ }^{*}\right)$ represent the observables for two physical systems, then $\left(\mathbb{Q}_{1} \otimes \mathbb{Q}_{2},{ }^{*}\right)$ represents the observables for the two systems combined. Let $\omega$ be a faithful state on $\left(Q_{1} \otimes Q_{2},{ }^{*}\right)$ so that $\left(Q_{1} \otimes Q_{2},{ }^{*}, \omega\right)$ becomes a probability algebra. Define the states $\omega_{1}, \omega_{2}$ on $\mathbb{Q}_{1}, \mathbb{Q}_{2}$, respectively, by $\omega_{1}\left(x_{1}\right)=\omega\left(x_{1} \otimes 1\right), \omega_{2}\left(x_{2}\right)=\omega\left(1 \otimes x_{2}\right)$. Then $\omega_{1}, \omega_{2}$ are faithful states called the reduced states. Conversely, if $\omega_{1}, \omega_{2}$ are faithful states on $\mathbb{Q}_{1}, \mathbb{Q}_{2}$, respectively, then we define the faithful state $\omega_{1} \otimes \omega_{2}$ on $\left(\mathbb{Q}_{1} \otimes \mathbb{Q}_{2},{ }^{*}\right)$ by $\omega_{1} \otimes \omega_{2}\left(x_{1} \otimes x_{2}\right)=\omega_{1}\left(x_{1}\right) \omega_{2}\left(x_{2}\right)$. If $\omega=\omega_{1} \otimes \omega_{2}$ we say that $\left(\mathscr{Q}_{1},{ }^{*}\right)$, $\left(\mathbb{Q}_{2},{ }^{*}\right)$ are uncorrelated relative to $\omega$. Physically, this means that when the two 
component systems are combined they do not interact.

THEOREM 5. The following statements are equivalent: (a) $\left(\mathbb{Q}_{1},{ }^{*}\right),\left(\mathbb{Q}_{2},{ }^{*}\right)$ are uncorrelated relative to $\omega$; (b) $E\left(x_{1} \otimes x_{2} \mid \mathscr{Q}_{1} \otimes 1\right)=\omega_{2}\left(x_{2}\right) x_{1} \otimes 1$ for all $x_{1} \in$ $\mathbb{Q}_{1}, x_{2} \in \mathbb{Q}_{2} ;(c) E\left(x_{1} \otimes x_{2} \mid \mathbb{Q}_{1} \otimes 1\right)=f\left(x_{1}, x_{2}\right) x_{1} \otimes 1$ for all $x_{1} \in \mathbb{Q}_{1}, x_{2} \in$ $\mathbb{Q}_{2}$; (d) for any state $\nu$ on $\mathbb{Q}_{1} \otimes 1$ with $\nu<\omega \mid \mathbb{Q}_{1} \otimes 1$, the $\left(\mathbb{Q}_{1} \otimes 1, \omega\right)$ coarsegraining of $\nu_{c}$ of $\nu$ exists and $\nu_{c}=\nu \otimes \omega_{2}$ (identity $\mathbb{Q}_{1} \otimes 1$ and $\mathbb{Q}_{1}$ in the natural way).

Proof. (a) $\Rightarrow$ (b) If (a) holds then $\omega_{2}\left(x_{2}\right) x_{1} \otimes 1 \in \mathbb{Q}_{1} \otimes 1$ and for any $y_{1} \in \mathbb{Q}_{1}$ we have

$$
\begin{aligned}
\omega\left[\left(y_{1} \otimes 1\right) \omega_{2}\left(x_{2}\right) x_{1} \otimes 1\right] & =\omega_{2}\left(x_{2}\right) \omega\left(y_{1} x_{1} \otimes 1\right) \\
& =\omega_{1}\left(y_{1} x_{1}\right) \omega_{2}\left(x_{2}\right)=\omega_{1} \otimes \omega_{2}\left(y_{1} x_{1} \otimes x_{2}\right) \\
& =\omega\left[\left(y_{1} \otimes 1\right)\left(x_{1} \otimes x_{2}\right)\right] .
\end{aligned}
$$

Hence $E\left(x_{1} \otimes x_{2} \mid \mathscr{Q}_{1} \otimes 1\right)=\omega_{2}\left(x_{2}\right) x_{1} \otimes 1$.

(b) $\Rightarrow$ (c) is trivial.

(c) $\Rightarrow$ (d) Suppose (c) holds. Then for $x_{1}, y_{1} \in \mathbb{Q}_{1}, x_{2} \in \mathbb{Q}_{2}$ we have

$$
\begin{aligned}
\omega\left(y_{1} x_{1} \otimes x_{2}\right) & =\omega\left[\left(y_{1} \otimes 1\right)\left(x_{1} \otimes x_{2}\right)\right] \\
& =\omega\left[\left(y_{1} \otimes 1\right) E\left(x_{1} \otimes x_{2} \mid \mathcal{Q}_{1} \otimes 1\right)\right] \\
& =f\left(x_{1}, x_{2}\right) \omega\left(y_{1} x_{1} \otimes 1\right)=f\left(x_{1}, x_{2}\right) \omega_{1}\left(y_{1} x_{1}\right) .
\end{aligned}
$$

Letting $y_{1}=1$ gives $f\left(x_{1}, x_{2}\right)=\omega\left(x_{1} \otimes x_{2}\right) / \omega_{1}\left(x_{1}\right)$ and letting $x_{1}=1$ gives

$$
\omega\left(y_{1} \otimes x_{2}\right)=\omega\left(1 \otimes x_{2}\right) \omega_{1}\left(y_{1}\right)=\omega_{1}\left(y_{1}\right) \omega_{2}\left(x_{2}\right) \text {. }
$$

Hence $\omega=\omega_{1} \otimes \omega_{2}$ and $\left(\mathscr{Q}_{1},{ }^{*}\right),\left(\mathbb{Q}_{2},{ }^{*}\right)$ are uncorrelated relative to $\omega$. Let $T=d \nu / d \omega \mid \mathbb{Q}_{1} \otimes 1$. Then $T \otimes I$ is a nonnegative extension of $T$ to $\mathbb{Q}_{1} \otimes \mathbb{Q}_{2}$. We now show that $T \otimes I \in \pi\left(\mathbb{Q}_{1} \otimes \mathbb{Q}_{2}\right)^{c}$. Indeed,

$$
\begin{aligned}
\langle T \otimes I \pi & \left.\left(x_{1} \otimes x_{2}\right) y_{1} \otimes y_{2}, z_{1} \otimes z_{2}\right\rangle \\
& =\left\langle T x_{1} y_{1} \otimes x_{2} y_{2}, z_{1} \otimes z_{2}\right\rangle \\
& =\left\langle T x_{1} y_{1}, z_{1}\right\rangle\left\langle x_{2} y_{2}, z_{2}\right\rangle=\left\langle T y_{1}, x_{1}^{*} z_{1}\right\rangle\left\langle y_{2}, x_{2}^{*} z_{2}\right\rangle \\
& =\left\langle T y_{1} \otimes y_{2}, x_{1}^{*} z_{1} \otimes x_{2}^{*} z_{2}\right\rangle \\
& =\left\langle T \otimes I y_{1} \otimes y_{2},\left(x_{1} \otimes x_{2}\right)^{*}\left(z_{1} \otimes z_{2}\right)\right\rangle \\
& =\left\langle T \otimes I y_{1} \otimes y_{2}, \pi\left[\left(x_{2} \otimes x_{2}\right)^{*}\right] z_{1} \otimes z_{2}\right\rangle .
\end{aligned}
$$

It follows from Theorem 3(c) that $\nu_{c}$ exists. Now $\nu_{c}=\nu \otimes \omega_{2}$ since from (b) we have

$$
\begin{aligned}
\nu_{c}\left(x_{1} \otimes x_{2}\right) & =\nu\left[E\left(x_{1} \otimes x_{2} \mid \mathcal{Q}_{1} \otimes 1\right)\right]=\nu\left[\omega_{2}\left(x_{2}\right) x_{1} \otimes 1\right] \\
& =\omega_{2}\left(x_{2}\right) \nu\left(x_{1}\right)=\nu \otimes \omega_{2}\left(x_{1} \otimes x_{2}\right) .
\end{aligned}
$$

(d) $\Rightarrow$ (a) If (d) holds then $\omega=\omega_{1_{c}}=\omega_{1} \otimes \omega_{2}$. 
Theorem 5(d) has the following physical interpretation. Suppose $\left(\mathbb{Q}_{2},{ }^{*}\right)$ represents a physical system whose state we want to determine. We let $\left(\mathbb{Q}_{2},{ }^{*}\right)$ interact with a measuring device represented by $\left(\mathscr{Q}_{1},{ }^{*}\right)$ to get a compound system $\left(\mathbb{Q}_{1} \otimes \mathbb{Q}_{2},{ }^{*}\right)$. Let $\omega$ be an a priori state giving a probability algebra $\left(\mathbb{Q}_{1} \otimes \mathbb{Q}_{2},{ }^{*}, \omega\right)$. A measurement of $\left(\mathbb{Q}_{1},{ }^{*}\right)$ gives a state $\nu$ on $\mathbb{Q}_{1} \otimes 1$ such that $\nu<\omega \mid \mathscr{Q}_{1} \otimes 1$. The $\left(Q_{1} \otimes 1, \omega\right)$ coarse-graining $\nu_{c}$ of $\nu$ gives the "most likely" state of the compound system. The reduced state $\nu_{c^{2}}$ gives the "most likely" state of the physical system $\left(\mathbb{Q}_{2},{ }^{*}\right)$. If $\left(\mathbb{Q}_{1},{ }^{*}\right),\left(\mathbb{Q}_{2},{ }^{*}\right)$ are uncorrelated relative to $\omega$, then we have seen that $\nu_{c}=\nu \otimes \omega_{2}$ so that $\nu_{c^{2}}=\omega_{2}$. Hence, in this case, the measurement gives no additional information about the state of the system.

4. The unbounded commutant. We have seen the importance of the unbounded commutant $\pi(\mathscr{Q})^{c}$ in the previous sections. In this section we characterize $\pi(\mathscr{Q})^{c}$.

Let $(Q, *, \omega)$ be a probability algebra. For $y \in D\left(\pi^{*}\right)$ define $\pi^{c}(y) \in$ $L(\mathbb{Q})$ by $\pi^{c}(y) x=\pi^{*}(x) y$ for every $x \in \mathbb{Q}$. A sequence of operators $C_{i} \in$ $L(\mathbb{Q})$ converges weakly to $C \in L(\mathbb{Q})$ if $\left\langle C_{i} x, y\right\rangle \rightarrow\langle C x, y\rangle$ for every $x$, $y \in \mathbb{Q}$.

THEOREM 6. $\pi^{c}$ is a weakly continuous linear bijection from $D\left(\pi^{*}\right)$ onto $\pi(\mathbb{Q})^{c}$.

Proof. Clearly, $\pi^{c}$ is linear. To show that $\pi^{c}$ maps $D\left(\pi^{*}\right)$ into $\pi(\mathscr{Q})^{c}$, for $y \in D\left(\pi^{*}\right), x, z, u \in \mathbb{Q}$ we have

$$
\begin{aligned}
\left\langle\pi^{c}(y) \pi(x) z, u\right\rangle & =\left\langle\pi^{c}(y) x z, u\right\rangle=\left\langle\pi^{*}(x z) y, u\right\rangle \\
& =\left\langle\pi^{*}(x) \pi^{*}(z) y, u\right\rangle=\left\langle\pi^{*}(z) y, \pi\left(x^{*}\right) u\right\rangle \\
& =\left\langle\pi^{c}(y) z, \pi\left(x^{*}\right) u\right\rangle .
\end{aligned}
$$

To show that $\pi^{c}$ is surjective, let $C \in \pi(\mathbb{Q})^{c}$. Then $C 1 \in D\left(\pi^{*}\right)$, and for every $x \in \mathbb{Q}$ we have

$$
\pi^{c}(C 1) x=\pi^{*}(x) C 1=C \pi(x) 1=C x .
$$

To show that $\pi^{c}$ is injective, suppose $y, y_{1} \in D\left(\pi^{*}\right)$ and $\pi^{c}(y)=\pi^{c}\left(y_{1}\right)$. Then

$$
y=\pi^{*}(1) y=\pi^{c}(y) 1=\pi^{c}\left(y_{1}\right) 1=\pi^{*}(1) y_{1}=y_{1} .
$$

To show that $\pi^{c}$ is weakly continuous, suppose $y_{i}, y \in D\left(\pi^{*}\right)$ and $y_{i} \rightarrow y$ in norm. Then for every $x, z \in \mathbb{Q}$ we have

$$
\begin{aligned}
\lim \left\langle\pi^{c}\left(y_{i}\right) x, z\right\rangle & =\lim \left\langle\pi^{*}(y) y_{i}, z\right\rangle=\lim \left\langle y_{i}, \pi\left(x^{*}\right) z\right\rangle \\
& =\left\langle y, \pi\left(x^{*}\right) z\right\rangle=\left\langle\pi^{*}(x) y, z\right\rangle=\left\langle\pi^{c}(y) x, z\right\rangle .
\end{aligned}
$$

The above theorem gives a characterization of $\pi(\mathscr{Q})^{c}$ in the sense that $\pi(\mathbb{Q})^{c}=\pi^{c}\left[D\left(\pi^{*}\right)\right]$. We shall later give a similar characterization of $\pi(\mathbb{Q})^{\prime}$, 
but first we shall analyze the structure of $\pi(\mathbb{Q})^{c}$. Let $M$ be a dense subspace of a Hilbert space $H$ and let $S \subseteq L(M)$. We say that $S$ is weakly closed if for any net $C_{\alpha} \in S$ which converges weakly to an operator $C \in L(M)$ we have $C \in S$. We call $S$ an op-algebra if for all $A, B \in S, A: M \rightarrow M$ and $A B \in S$, $(\alpha A+B) \in S$ for all $\alpha \in \mathrm{C}$. For $A \in S$, denote the adjoint of $A$ by $A^{\dagger}$, and if $D\left(A^{\dagger}\right) \supseteq M$ define $A^{*}=A^{\dagger} \mid M$. We call an op-algebra $S$ an op -algebra if $I \mid M \in S$ and if for all $A \in S$ we have $D\left(A^{\dagger}\right) \supseteq M$ and $A^{*} \in S$. If $S$ is an op*-algebra, it is easy to see that $^{*}$ is an involution on $S$ and hence $S$ is a *-algebra. A *-representation $\pi$ of a ${ }^{*}$-algebra $\left(\mathcal{Q},{ }^{*}\right)$ is selfadjoint if $\pi=\pi^{*}$ (i.e. $\left.D(\pi)=D\left(\pi^{*}\right)\right)[13]$.

Now let $\left(\mathscr{Q},{ }^{*}, \omega\right)$ be a probability algebra. It is easy to see that $\pi(\mathscr{Q})^{c}$ is a weakly closed linear manifold with identity, in $L(\mathscr{Q})$. In general, $\pi(\mathbb{Q})^{c}$ need not be an op-algebra, let alone an op*-algebra. Theorem 8, to follow, characterizes when these circumstances hold.

LEMMA 7. Let $\left(\mathbb{Q},{ }^{*}, \omega\right)$ be a probability algebra. If there exists a map $x \rightarrow x^{b}$ on $\mathbb{Q}$ such that $\omega\left(y^{*} x\right)=\omega\left(x y^{b}\right)$ for all $x, y \in \mathbb{Q}$ then ${ }^{b}$ is unique, is an involution, $\left\langle x^{*}, y\right\rangle=\left\langle y^{b}, x\right\rangle$ and $\langle x y, z\rangle=\left\langle x, z y^{b}\right\rangle$ for all $x, y, z \in \mathbb{Q}$.

Proof. To show ${ }^{b}$ is unique, suppose ' is another map such that $\omega\left(y^{*} x\right)=$ $\omega\left(x y^{\prime}\right)$ for all $x, y \in \mathbb{Q}$. Then for all $x, y \in \mathbb{Q}$ we have

$$
\left\langle y^{\prime}, x\right\rangle=\omega\left(x^{*} y^{\prime}\right)=\omega\left(x^{*} y^{b}\right)=\left\langle y^{b}, x\right\rangle,
$$

so $y^{\prime}=y^{b}$. That ${ }^{b}$ is an involution follows from the equations:

$$
\begin{gathered}
\begin{array}{c}
\omega\left[z(x+y)^{b}\right]=\omega\left[(x+y)^{*} z\right]=\omega\left(x^{*} z\right)+\omega\left(y^{*} z\right) \\
=\omega\left(z x^{b}\right)+\omega\left(z y^{b}\right)=\omega\left[z\left(x^{b}+y^{b}\right)\right]
\end{array} \\
\omega\left[z(x y)^{b}\right]=\omega\left[(x y)^{*} z\right]=\omega\left(y^{*} x^{*} z\right)=\omega\left(x^{*} z y^{b}\right)=\omega\left(z y^{b} x^{b}\right) \\
\begin{array}{c}
\omega\left[z(\alpha x)^{b}\right]=\omega\left[(\alpha x)^{*} z\right]=\omega\left(\alpha^{*} x^{*} z\right)=\omega\left(z \alpha^{*} x^{b}\right) \\
\omega\left(z x^{b b}\right)=\omega\left(x^{b *} z\right)=\omega\left[\left(z^{*} x^{b}\right)^{*}\right]=\left[\omega\left(z^{*} x^{b}\right)\right]^{*} \\
=\left[\omega\left(x^{*} z^{*}\right)\right]^{*}=\omega(z x) .
\end{array}
\end{gathered}
$$

Finally, we have

$$
\left\langle x^{*}, y\right\rangle=\omega\left(y^{*} x^{*}\right)=\omega\left(x^{*} y^{b}\right)=\left\langle y^{b}, x\right\rangle
$$

and

$$
\begin{aligned}
\langle x y, z\rangle & =\omega\left(z^{*} x y\right)=\omega\left(x y z^{b}\right)=\omega\left[x\left(z y^{b}\right)^{b}\right] \\
& =\omega\left[\left(z y^{b}\right)^{*} x\right]=\left\langle x, z y^{b}\right\rangle . \quad \square
\end{aligned}
$$

Suppose there exists an involution ${ }^{b}$ on the probability algebra $\left(Q,{ }^{*}, \omega\right)$ such that $\omega\left(y^{*} x\right)=\omega\left(x y^{b}\right)$ for all $x, y \in \mathbb{Q}$. For $x \in \mathbb{Q}$ define the operator 
$\rho(x) \in L(\mathbb{Q})$ by $\rho(x) y=y x$. It is easy to check that $\rho: \mathbb{Q} \rightarrow L(\mathbb{Q})$ is a b-antirepresentation of $\mathbb{Q}$.

THEOREM 8. Let $\left(\mathbb{Q},{ }^{*}, \omega\right)$ be a probability algebra.

(1) The following statements are equivalent:

(a) $\pi(\mathbb{Q})^{c}$ is an algebra;

(b) $C 1 \in \mathbb{Q}$ for every $C \in \pi(\mathbb{Q})^{c}$;

(c) for any $F<\omega$ there exists a $y \in \mathbb{Q}$ such that $F(x)=\langle x, y\rangle$;

(d) $\pi$ is selfadjoint.

(2) $\pi(Q)^{c}$ is $a^{*}$-algebra if and only if $\pi$ is selfadjoint and there exists an involution ${ }^{b}$ on $\mathbb{Q}$ such that $\omega\left(y^{*} x\right)=\omega\left(x y^{b}\right)$ for all $x, y \in \mathbb{Q}$.

(3) If $\pi(\mathbb{Q})^{c}$ is $a^{*}$-algebra, then $\rho$ is a weakly continuous b-anti-isomorphism of $\mathbb{Q}$ onto $\pi(\mathbb{Q})^{c}$.

Proof. (1) (a) $\Rightarrow$ (b) is trivial. (b) $\Rightarrow$ (c) Suppose (b) holds and $F<\omega$. By Theorem 1 , there exists a $C \in \pi(\mathbb{Q})^{c}$ such that $F(x)=\left[\omega\left(C x^{*}\right)\right]^{*}$ for every $x \in \mathbb{Q}$. If $y=C 1 \in \mathbb{Q}$, then for all $x \in \mathbb{Q}$ we have

$$
F(x)=\left[\omega\left(C x^{*}\right)\right]^{*}=\left\langle C x^{*}, 1\right\rangle^{*}=\left\langle 1, C x^{*}\right\rangle=\langle x, y\rangle .
$$

(c) $\Rightarrow$ (d) Suppose (c) holds and $z \in D\left(\pi^{*}\right)$. As in the proof of Theorem 4, the linear functional $F(x)=\langle x, z\rangle$ is absolutely continuous. Hence, there exists a $z^{\prime} \in \mathbb{Q}$ such that $\langle x, z\rangle=\left\langle x, z^{\prime}\right\rangle$ for all $x \in \mathbb{Q}$. Thus $z=z^{\prime} \in \mathbb{Q}$, so $D\left(\pi^{*}\right)=\mathbb{Q}=D(\pi)$ and $\pi$ is selfadjoint.

(d) $\Rightarrow$ (a) Suppose (d) holds. Then $C: \mathbb{Q} \rightarrow \mathbb{Q}$ for every $C \in \pi(\mathbb{Q})^{c}$. Furthermore, if $A, B \in \pi(\mathbb{Q})^{c}$ then for all $x \in \mathbb{Q}$ we have

$$
A B \pi(x)=A \pi(x) B=\pi(x) A B
$$

so $\pi(\mathscr{Q})^{c}$ is an algebra.

(2) Suppose $\pi(\mathbb{Q})^{c}$ is a ${ }^{*}$-algebra. By (1), $\pi$ is selfadjoint. If $C \in \pi(\mathscr{Q})^{c}$, then $C^{*}=C^{\dagger} \mid \mathbb{Q} \in \pi(\mathbb{Q})^{c}$ so $C^{*}: \mathbb{Q} \rightarrow \mathbb{Q}$. For $y \in \mathbb{Q}$, by Theorem $6, \pi^{c}(y) \in$ $\pi(\mathbb{Q})^{c}$ so $y^{b} \equiv\left[\pi^{c}(y)\right]^{*} 1 \in \mathbb{Q}$. For all $x \in \mathbb{Q}$, we have

$$
\begin{aligned}
\omega\left(x y^{b}\right) & =\left\langle y^{b}, x^{*}\right\rangle=\left\langle\left[\pi^{c}(y)\right]^{*} 1, x^{*}\right\rangle=\left\langle 1, \pi^{c}(y) x^{*}\right\rangle \\
& =\left\langle 1, \pi^{*}\left(x^{*}\right) y\right\rangle=\left\langle 1, x^{*} y\right\rangle=\langle x, y\rangle=\omega\left(y^{*} x\right) .
\end{aligned}
$$

Applying Lemma 7, b is an involution on $\mathcal{Q}$. Conversely, suppose $\pi$ is selfadjoint and there exists an involution $b$ on $\mathbb{Q}$ such that $\omega\left(y^{*} x\right)=\omega\left(x y^{b}\right)$ for all $x, y \in \mathbb{Q}$. Then by (1), $\pi(\mathbb{Q})^{c}$ is an algebra. If $C \in \pi(\mathbb{Q})^{c}$, then for all $x, y \in \mathbb{Q}$ we have

$$
\langle C y, x\rangle=\left\langle C 1, y^{*} x\right\rangle=\left\langle(C 1) x^{b}, y^{*}\right\rangle=\left\langle y, x(C 1)^{b}\right\rangle .
$$

Hence $\mathscr{Q} \subseteq D\left(C^{\dagger}\right)$ and $C^{\dagger} \mid \mathbb{Q}=\pi^{c}\left[(C 1)^{b}\right] \in \pi^{c}(\mathscr{Q})=\pi(\mathbb{Q})^{c}$. Thus $\pi(\mathbb{Q})^{c}$ is a *-algebra.

(3) If $\pi(Q)^{c}$ is a ${ }^{*}$-algebra, then $\pi$ is selfadjoint so $\rho(x)=\pi^{c}(x)$ for all $x \in \mathbb{Q}$. It follows from Theorem 6 that $\rho: \mathbb{Q} \rightarrow \pi(\mathbb{Q})^{c}$ is a weakly continuous, 
linear bijection and it is clear that $\rho$ is an anti-isomorphism. Finally, for all $x$, $y \in \mathbb{Q}$ we have

$$
\rho\left(x^{b}\right) y=y x^{b}=y\left\{\left[\pi^{c}(x)\right]^{*} 1\right\}=\left[\pi^{c}(x)\right]^{*} y=[\rho(x)]^{*} y .
$$

Notice that Theorem $8(3)$ gives a characterization of $\pi(\mathscr{Q})^{c}$ of the form $\pi(\mathbb{Q})^{c}=\rho(\mathbb{Q})$. We shall later discuss a similar characterization of $\pi(\mathbb{Q})^{\prime}$.

5. The commutant. Let $\left(\mathscr{Q},{ }^{*}, \omega\right)$ be a probability algebra and let $\mathbb{Q}_{0}=\{y$ $\in \mathbb{Q}: \mathbb{Q} \ni x \rightarrow \pi(x) y$ is bounded $\}, D\left(\pi^{*}\right)_{0}=\left\{y \in D\left(\pi^{*}\right): \mathbb{Q} \ni x \rightarrow\right.$ $\pi^{*}(x) y$ is bounded $\}$.

Of course, $\mathbb{Q}_{0} \subseteq D\left(\pi^{*}\right)_{0}$. If $y \in D\left(\pi^{*}\right)_{0}$, we define $\pi^{\prime}(y)$ to be the unique bounded operator on $H$ which satisfies $\pi^{\prime}(y) x=\pi^{*}(x) y$ for all $y \in \mathbb{Q}$.

LEMMA 9. $\pi^{\prime}$ is a weakly continuous, linear bijection from $D\left(\pi^{*}\right)_{0}$ onto $\pi(\mathbb{Q})^{\prime}$.

Proof. This is similar to the proof of Theorem 6.

If $\pi$ is selfadjoint we can, of course, replace $D\left(\pi^{*}\right)_{0}$ by $\mathbb{Q}_{0}$ in Lemma 9 . This selfadjointness condition, which was also used in $\$ 4$, is a very strong requirement which eliminates many cases of interest. For this reason, we shall impose the weaker, more reasonable, condition that $\pi$ is essentially selfadjoint; that is, the closure $\bar{\pi}$ of $\pi$ is selfadjoint. Furthermore, one of the difficulties with $Q_{0}$ and $D\left(\pi^{*}\right)_{0}$ is that they might not be closed under the * operation. This difficulty is circumvented by introducing a class of probability algebras (which we call closable) in which ${ }^{*}$ is a closable operator.

For $x, y \in \mathbb{Q}$ define the seminorms $\|x\|_{y}=\|y x\|$. The induced topology on $\mathbb{Q}$ is the topology generated by the collection of seminorms $\left\{\|x\|_{y}: y \in \mathbb{Q}\right\}$. This is the weakest topology on $\mathbb{Q}$ in which $\pi(x)$ is bounded for every $x \in \mathbb{Q}$. Let $\hat{\mathcal{Q}}$ be the completion of $\mathscr{Q}$ relative to the induced topology. Then $\mathbb{Q} \subseteq \hat{\mathscr{Q}} \subseteq H$ and $\hat{\mathcal{Q}}$ is a dense subspace of $H$. Since $\pi(x)$ is continuous on $\mathbb{Q}$ (in the induced topology), $\pi(x)$ has a unique continuous (in the induced topology) extension to $\hat{\mathscr{Q}}$ which we denote by $\bar{\pi}(x)$. We call $\bar{\pi}$ the closure of $\pi$. It can be shown that $\bar{\pi}$ is a ${ }^{*}$-representation of $\mathcal{Q}$ with domain $D(\bar{\pi})=\hat{\mathbb{Q}}$, that $\bar{\pi} \subseteq \pi^{*},(\bar{\pi})^{*}=\pi^{*}$, and $\bar{\pi}(\mathbb{Q})^{\prime}=\pi(\mathbb{Q})^{\prime}[13]$. Thus $\pi$ is essentially selfadjoint if and only if $\bar{\pi}=\pi^{*}$. In general, although $\pi(\mathbb{Q})^{\prime}$ is a weakly closed, *-closed, linear manifold in $L(H), \pi(\mathbb{Q})^{\prime}$ need not be a von Neumann algebra [13]. If, however, $\pi$ is essentially selfadjoint, then it is immediate that $\pi(\mathbb{Q})^{\prime}$ is a von Neumann algebra.

LEMMA 10. (1) The following statements are equivalent:

(a) $\pi$ is essentially selfadjoint;

(b) $C 1 \in \hat{\mathbb{Q}}$ for every $C \in \pi(\mathbb{Q})^{c}$;

(c) for any $F<\omega$ there exists a $y \in \hat{\mathscr{Q}}$ such that $F(x)=\langle x, y\rangle$, for all $x \in \mathbb{Q}$.

(2) If $\pi(x)$ is a bounded operator for every $x \in \mathbb{Q}$, then $\pi$ is selfadjoint. 
Proof. (1) Similar to Theorem 8(1). (2) If the $\pi(x)$ are bounded then $\hat{\mathbb{Q}}=H$ so $D(\bar{\pi})=D\left(\pi^{*}\right)=H$.

A probability algebra $\left(\mathbb{Q},{ }^{*}, \omega\right)$ is closable if for any sequence $x_{i} \in \mathbb{Q}$ which satisfies $\lim \omega\left(x_{i}^{*} x_{i}\right)=0$ and $\lim \omega\left(x_{i} x_{i}^{*}\right)=\lim _{i, j} \operatorname{Re} \omega\left(x_{i} x_{j}^{*}\right)$ we have $\lim \omega\left(x_{i} x_{i}^{*}\right)=0$. A lip algebra is a ${ }^{*}$-algebra $\left(\mathbb{Q},{ }^{*}\right)$ with an inner product $\langle\cdot, \cdot\rangle$ satisfying:

(5.1) $\langle 1,1\rangle=1,\left\langle x^{*} x, 1\right\rangle=\langle x, x\rangle$ for all $x \in \mathbb{Q}$;

(5.2) $x \mapsto x^{*}$ is closable in the completion $H$ of $\mathscr{Q}$.

A lip algebra is more general than a Tomita left Hilbert algebra [17] in the sense that left multiplication is not assumed to be continuous; it is less general in the sense that an identity is assumed. The next lemma shows that there is essentially no difference between a closable probability algebra and a lip algebra.

LEMMA 11. (1) Let $\left(\mathscr{Q},{ }^{*}, \omega\right)$ be a closable probability algebra. If we define $\langle x, y\rangle=\omega\left(y^{*} x\right)$, then $\left(\mathbb{Q},{ }^{*},\langle\cdot, \cdot\rangle\right)$ is a lip algebra.

(2) Let $\left(\mathbb{Q},{ }^{*},\langle\cdot, \cdot\rangle\right)$ be a lip algebra. If we define $\omega(x)=\langle x, 1\rangle$, then $\left(\mathscr{Q},{ }^{*}, \omega\right)$ is a closable probability algebra such that $\langle x, y\rangle=\omega\left(y^{*} x\right)$.

(3) In any lip algebra, $\langle x y, z\rangle=\langle y, x * z\rangle$.

Proof. (1) Clearly $\langle\cdot, \cdot\rangle$ is an inner product and (5.1) holds. To show $x \rightarrow x^{*}$ is closable, suppose $x_{i} \rightarrow 0$ and $x_{i}^{*}$ is Cauchy. Then $\omega\left(x_{i}^{*} x_{i}\right)=\left\|x_{i}\right\|^{2}$ $\rightarrow 0$ and

$$
\begin{aligned}
\omega\left(x_{i} x_{i}^{*}\right)+ & \omega\left(x_{j} x_{j}^{*}\right)-2 \operatorname{Re} \omega\left(x_{i} x_{j}^{*}\right) \\
& =\left\langle x_{i}^{*}, x_{i}^{*}\right\rangle+\left\langle x_{j}^{*}, x_{i}^{*}\right\rangle-2 \operatorname{Re}\left\langle x_{j}^{*}, x_{i}^{*}\right\rangle=\left\|x_{i}^{*}-x_{j}^{*}\right\|^{2} \rightarrow 0 .
\end{aligned}
$$

Since $x_{i}^{*}$ converges in $H, \omega\left(x_{i} x_{i}^{*}\right)=\left\|x_{i}^{*}\right\|^{2}$ converges. Hence $\operatorname{Re} \omega\left(x_{i} x_{j}^{*}\right)$ converges and $\lim _{i, j} \operatorname{Re} \omega\left(x_{i} x_{j}^{*}\right)=\lim \omega\left(x_{i} x_{i}^{*}\right)$. It follows that $\left\|x_{i}^{*}\right\|^{2}=\omega\left(x_{i} x_{i}^{*}\right)$ $\rightarrow 0$, so $x_{i}^{*} \rightarrow 0$.

(2) Since $\omega\left(x^{*} x\right)=\left\langle x^{*} x, 1\right\rangle=\langle x, x\rangle=\|x\|^{2}$, we see that $\omega$ is a faithful state. The other condition for a closable probability algebra follows by reversing the steps in (1). That $\langle x, y\rangle=\omega\left(y^{*} x\right)$ follows from $\|x\|^{2}=\omega\left(x^{*} x\right)$ and the polarization equation

$$
\langle x, y\rangle=\frac{1}{2}\left[i\|x+i y\|^{2}+\|x+y\|^{2}-(1+i)\left(\|x\|^{2}+\|y\|^{2}\right)\right] .
$$

(3) This follows from (2).

In the remainder of this section, $\left(\mathscr{Q},{ }^{*}, \omega\right)$ will be a closable probability algebra. If $S$ denotes the closure of ${ }^{*}$, then $S$ is a closed conjugate-linear operator with $D(S) \supseteq \mathbb{Q}$. The adjoint $S^{*}$ of $S$ is a closed, densely defined, conjugate-linear operator and $\langle S x, y\rangle=\left\langle S^{*} y, x\right\rangle$ for all $x \in D(S), y \in$ $D\left(S^{*}\right)$.

Lemma 12. $D(S)$ is invariant under $S$ and $S^{2} x=x$ for every $x \in D(S)$. 
$D\left(S^{*}\right)$ is invariant under $S^{*}$ and $S^{* 2} x=x$ for every $x \in D\left(S^{*}\right)$.

Proof. Let $x \in D(S), y \in D\left(S^{*}\right)$. Since $S$ is the closure of ${ }^{*}$ and $D\left(^{*}\right)=$ $\mathbb{Q}$, there exists a sequence $x_{i} \in \mathbb{Q}$ such that $x_{i} \rightarrow x$ and $S x_{i} \rightarrow S x$. Hence

$$
\begin{aligned}
\left\langle S^{*} y, S x\right\rangle & =\lim \left\langle S^{*} y, S x_{i}\right\rangle=\lim \left\langle S^{*} y, x_{i}^{*}\right\rangle \\
& =\lim \left\langle S x_{i}^{*}, y\right\rangle=\lim \left\langle x_{i}, y\right\rangle=\langle x, y\rangle .
\end{aligned}
$$

Thus $S x \in D(S)$ and $S^{2} x=x$. A similar argument holds for $S^{*}$.

Let $\mathbb{Q}^{\prime}=\left\{y \in D\left(S^{*}\right) \cap D\left(\pi^{*}\right): \mathbb{Q} \in x \rightarrow \pi^{*}(x) y\right.$ is bounded $\}$. For $y \in$ $\mathbb{Q}^{\prime}$, let $\pi^{\prime}(y)$ be the unique bounded operator on $H$ satisfying $\pi^{\prime}(y) x=$ $\pi^{*}(x) y$ for all $x \in \mathbb{Q}$.

LEMMA 13. $y \in \mathbb{Q}^{\prime}$ if and only if there exist a bounded operator $A$ and $a$ $y_{1} \in D\left(\pi^{*}\right)$ such that $A x=\pi^{*}(x) y, A^{*} x=\pi^{*}(x) y_{1}$ for every $x \in \mathbb{Q}$. In this case $y_{1}=S^{*} y, \pi^{\prime}(y)=A$.

Proof. Suppose there exist an $A$ and $y_{1} \in D\left(\pi^{*}\right)$. For any $x \in \mathbb{Q}$ we have

$$
\begin{aligned}
\left\langle x^{*}, y\right\rangle & =\left\langle\pi\left(x^{*}\right) 1, y\right\rangle=\left\langle 1, \pi^{*}(x) y\right\rangle \\
& =\langle 1, A x\rangle=\left\langle A^{*} 1, x\right\rangle=\left\langle y_{1}, x\right\rangle .
\end{aligned}
$$

Hence $y$ is in the domain of the adjoint of *. But the adjoint of a closable operator equals the adjoint of its closure, so $y \in D\left(S^{*}\right)$ and $S^{*} y=y_{1}$. Also $x \rightarrow \pi(x) y$ is bounded on $\mathbb{Q}$ and $\pi^{\prime}(y)=A$. Conversely, suppose $y \in \mathbb{Q}^{\prime}$ and let $A=\pi^{\prime}(y), y_{1}=S^{*} y$. Then $A x=\pi^{\prime}(y) x=\pi^{*}(x) y$ and for every $x$, $z \in \mathbb{Q}$ we have

$$
\begin{aligned}
\left\langle\pi\left(x^{*}\right) z, y_{1}\right\rangle & =\left\langle x^{*} z, S^{*} y\right\rangle=\left\langle y, z^{*} x\right\rangle=\left\langle y, \pi\left(z^{*}\right) x\right\rangle \\
& =\left\langle\pi^{*}(z) y, x\right\rangle=\langle A z, x\rangle=\left\langle z, A^{*} x\right\rangle .
\end{aligned}
$$

Hence $y_{1} \in D\left(\pi^{*}\right)$ and $\pi^{*}\left(y_{1}\right)=A^{*} x$ for all $x \in \mathbb{Q}$.

TheOREM 14. Let $(Q, *, \omega)$ be a closable probability algebra for which $\pi$ is essentially selfadjoint. Then $\mathbb{Q}^{\prime}$ is a $a^{*}$-algebra under the product $y_{1}^{\circ} y_{2}=$ $\pi^{\prime}\left(y_{1}\right) y_{2}$ and the involution $y^{*}=S^{*} y$. Furthermore, $\pi^{\prime}$ is $a^{*}$-isomorphism of $\mathbb{Q}^{\prime}$ onto $\pi(\mathbb{Q})^{\prime}$.

Proof. That $\pi^{\prime}$ is an injective map from $\mathbb{Q}^{\prime}$ to $\pi(\mathscr{Q})^{\prime}$ is similar to the proof in Theorem 6. To show that $\pi^{\prime}$ is surjective, let $A \in \pi(\mathbb{R})^{\prime}$. Then for all $x \in \mathbb{Q}$ we have

$$
\left\langle x^{*}, A 1\right\rangle=\left\langle A^{*} x^{*}, 1\right\rangle=\left\langle A^{*} 1, x\right\rangle .
$$

Hence $A 1 \in D\left(S^{*}\right)$ and $S^{*}(A 1)=A^{*} 1$. We conclude that $A 1 \in \mathbb{Q}^{\prime}$. Since $A x=\pi^{*}(x) A 1, A^{*} x=\pi^{*}(x) A^{*} 1$ for all $x \in \mathbb{Q}$, it follows from Lemma 13 that $\pi^{\prime}(A 1)=A$ so $\pi^{\prime}$ is surjective. If $y \in \mathbb{Q}^{\prime}$, then by Lemma $13, S^{*} y \in \mathbb{Q}^{\prime}$ and for all $x \in \mathbb{Q}$ we have

$$
\pi^{\prime}\left(S^{*} y\right) x=\pi^{*}(x) S^{*} y=A^{*} x=\pi^{\prime}(y)^{*} x .
$$


Furthermore, if $x \in \mathbb{Q}$ and $y_{1}, y_{2} \in \mathbb{Q}^{\prime}$, then

$$
\begin{aligned}
\pi^{\prime}\left(y_{1}\right) \pi^{\prime}\left(y_{2}\right) x & =\pi^{\prime}\left(y_{1}\right) \pi^{*}(x) y_{2}=\pi^{\prime}\left(y_{1}\right) \bar{\pi}(x) y_{2} \\
& =\pi^{*}(x) \pi^{\prime}\left(y_{1}\right) y_{2}
\end{aligned}
$$

and

$$
\begin{aligned}
\pi^{\prime}\left(y_{2}\right)^{*} \pi^{\prime}\left(y_{1}\right)^{*} x & =\pi^{\prime}\left(S^{*} y_{2}\right) \pi^{\prime}\left(S^{*} y_{1}\right) x=\pi^{\prime}\left(S^{*} y_{2}\right) \pi^{*}(x) S^{*} y_{1} \\
& =\pi^{*}(x) \pi^{\prime}\left(S^{*} y_{2}\right) S^{*} y_{1} .
\end{aligned}
$$

By Lemma 13, $\pi^{\prime}\left(y_{1}\right) y_{2} \in \mathbb{Q}^{\prime}, S^{*} \pi^{\prime}\left(y_{1}\right) y_{2}=\pi^{\prime}\left(S^{*} y_{2}\right) S^{*} y_{1}$, and $\pi^{\prime}\left[\pi^{\prime}\left(y_{1}\right) y_{2}\right]$ $=\pi^{\prime}\left(y_{1}\right) \pi^{\prime}\left(y_{2}\right)$. It is now easy to verify that $\mathscr{Q}$ is an algebra under the product $y_{1} \circ y_{2}=\pi^{\prime}\left(y_{1}\right) y_{2}$ and that $\pi^{\prime}$ is an isomorphism from $\mathbb{Q}^{\prime}$ onto $\pi(\mathbb{Q})^{\prime}$. The operation $y^{*}=S^{*} y$ is an involution since $S^{*}$ is conjugate linear, $S^{* 2}=I$, and

$$
S^{*}\left(y_{1} \circ y_{2}\right)=S^{*} \pi^{\prime}\left(y_{1}\right) y_{2}=\pi^{\prime}\left(S^{*} y_{2}\right) S^{*} y_{1}=y_{2}^{*} \circ y_{1}^{*} .
$$

Finally, $\pi^{\prime}$ is a ${ }^{*}$-isomorphism by (5.3).

COROllary. $\mathbb{Q}^{\prime}$ is a Tomita left Hilbert algebra.

Proof. $\mathbb{Q}^{\prime}$ is a lip algebra, since for every $x \in \mathbb{Q}^{\prime}$ we have

$$
\begin{aligned}
\left\langle x^{*} \circ x, 1\right\rangle & =\left\langle\left(S^{*} x\right) \circ x, 1\right\rangle=\left\langle\pi^{\prime}\left(S^{*} x\right) x, 1\right\rangle \\
& =\left\langle\pi^{\prime}(x)^{*} x, 1\right\rangle=\left\langle x, \pi^{\prime}(x) 1\right\rangle=\langle x, x\rangle .
\end{aligned}
$$

Furthermore, left multiplication is bounded.

Our next result is analogous to a result of Tomita and Takesaki [17]. Our proof follows van Daele [3, p. 381] closely.

THEOREM 15. Let $\left(\mathcal{Q},{ }^{*}, \omega\right)$ be a closable probability algebra. Then there exist a bounded conjugate-linear operator $J: H \rightarrow H$ such that $J^{2}=I, J=J^{*}$ and $a$ positive selfadjoint (not necessarily bounded) operator $\Delta$ on $H$ satisfying:

(1) $\Delta=S^{*} S, \Delta^{-1}=S S^{*}$;

(2) $D(S)=D\left(\Delta^{1 / 2}\right), D\left(S^{*}\right)=D\left(\Delta^{-1 / 2}\right)$;

(3) $J \Delta J=\Delta^{-1}$ and $J f(\Delta) J=f^{*}\left(\Delta^{-1}\right)$ for every measurable function $f$ on $(0, \infty)$

(4) $S=J \Delta^{1 / 2}=\Delta^{-1 / 2} J, S^{*}=J \Delta^{-1 / 2}=\Delta^{1 / 2} J$.

Proof. Let $H^{*}$ be the Hilbert space consisting of the set $H$ with scalar multiplication $(\alpha, x) \rightarrow \alpha^{*} x$ and inner product $(x, y) \rightarrow\langle y, x\rangle$. Then $S$ : $D(S) \rightarrow H^{*}$ is a closed linear operator with dense domain. Hence $\Delta=S^{*} S$ is a nonnegative selfadjoint operator on $H$. By the polar decomposition theorem $\left[11\right.$, p. 282] there exists a partial isometry $J: H \rightarrow H^{*}$ such that $S=J \Delta^{1 / 2}$ and the initial domain of $J$ is $\overline{R\left(S^{*}\right)}$. Now the range of $S^{*}$ is dense since $x \in D\left(S^{*}\right)$ implies $x=S^{*}\left(S^{*} x\right)$, so $D\left(S^{*}\right)=R\left(S^{*}\right)$ (identifying $H$ and $\left.H^{*}\right)$, and thus $\overline{R\left(S^{*}\right)}=H^{*}$. Hence $J$ is unitary. Since $\left(S^{*} S\right)\left(S S^{*}\right)=I$ on $\mathbb{Q}$ 
we have $\Delta^{-1}=S S^{*}$ and $\Delta$ is positive selfadjoint. Since $S=S^{-1}$ we have $J \Delta^{1 / 2}=\Delta^{-1 / 2} J^{*}$ and $J \Delta^{1 / 2} J=\Delta^{-1 / 2}$. Also, $\Delta^{-1 / 2}=J^{2} J^{*} \Delta^{1 / 2} J$, and as $J^{2}$ is unitary and $J^{*} \Delta^{1 / 2} J$ is positive, it follows from the uniqueness of the polar decomposition of $\Delta^{-1 / 2}$ that $J^{2}=I$ and, hence, $J=J^{*}$. (1), (2), and (4) now follow. To prove (3), notice that $J \Delta J=\Delta^{-1}$. If $g(\lambda)=\Sigma c_{i} \lambda^{i}$ is a polynomial, then

$$
g\left(\Delta^{-1}\right)=\sum c_{i}(J \Delta J)^{i}=\sum c_{i} J \Delta^{i} J=J\left(\sum c_{i}^{*} \Delta^{i}\right) J=J g^{*}(\Delta) J .
$$

If $f$ is a measurable function on $(0, \infty)$, it follows that $J f(\Delta) J=f^{*}\left(\Delta^{-1}\right) . \quad \square$

Notice that $J$ satisfies the equations $\langle J x, y\rangle=\langle J y, x\rangle$ and $\langle J x, J y\rangle=$ $\langle y, x\rangle$. If $\pi(x)$ is bounded, then $\mathbb{Q}^{\prime}$ is dense in $H$ [17]. In general, we have the following result.

Corollary. Let $\left(\mathbb{Q},{ }^{*}, \omega\right)$ be a closable probability algebra in which $\bar{\pi}$ is selfadjoint. If $\mathbb{Q}^{\prime}$ is dense in $H$ then $\pi(\mathbb{Q})^{\prime \prime}=J \pi(\mathbb{Q})^{\prime} J$.

Proof. By Tomita and Takesaki [17], we have $J \pi^{\prime}\left(\mathbb{Q}^{\prime}\right) J=\pi^{\prime}\left(\mathbb{Q}^{\prime}\right)^{\prime}$. Hence

$$
\pi(\mathbb{Q})^{\prime \prime}=\pi^{\prime}\left(\mathbb{Q}^{\prime}\right)^{\prime}=J \pi^{\prime}\left(\mathbb{Q}^{\prime}\right) J=J \pi(\mathbb{Q})^{\prime} J \text {. }
$$

6. Symmetric probability algebras. Let $(\mathcal{Q}, \#, \omega)$ be a probability algebra. If there exists a map $x \rightarrow x^{b}$ on $\mathbb{Q}$ such that $\omega\left(y^{\sharp} x\right)=\omega\left(x y^{b}\right)$ for every $x, y \in$ $\mathcal{Q}$, then we call $(\mathcal{Q}, \#, \omega)$ a symmetric probability algebra. Theorem 8 gives a sufficient (but not necessary) condition for a probability algebra to be symmetric. A rip algebra is an algebra $Q$ with an identity 1 , an involution b, and an inner product $\langle\cdot, \cdot\rangle$ satisfying:

(6.1) $\left\langle x x^{b}, 1\right\rangle=\langle x, x\rangle,\langle 1,1\rangle=1$;

(6.2) $x \rightarrow x^{b}$ is closable on the completion $H$ of $\mathscr{Q}$.

LEMMA 16. (1) Let $(Q, \#, \omega)$ be a symmetric probability algebra and let $\langle x, y\rangle=\omega\left(y^{\sharp} x\right)$. Then ${ }^{b}$ is unique, $(\mathbb{Q}, \#,\langle\cdot, \cdot\rangle)$ is a lip algebra and $\left(\mathbb{Q},{ }^{b}\right.$, $\langle\cdot, \cdot\rangle)$ is a rip algebra. Furthermore, for every $x, y, z \in \mathbb{Q}$ we have

$$
\langle x y, z\rangle=\left\langle y, x^{\sharp} z\right\rangle=\left\langle x, z y^{b}\right\rangle \text {. }
$$

(2) Let $\mathbb{Q}$ be an algebra with an identity 1 , two involutions \#, product $\langle\cdot, \cdot\rangle$ which satisfies $\langle x, x\rangle=\left\langle x^{*} x, 1\right\rangle=\left\langle x x^{b}, 1\right\rangle$. Define $\omega(x)=$ $\langle x, 1\rangle$. Then $(\mathscr{Q}, \#, \omega)$ is a symmetric probability algebra and $\langle x, y\rangle=\omega\left(y^{\sharp} x\right)$.

(3) A symmetric probability algebra is closable.

(4) A closable probability algebra $\left(\mathcal{Q},{ }^{*}, \omega\right)$ is symmetric if and only if $\mathbb{Q} \subseteq D\left(S^{*}\right)$ and $S^{*}: \mathbb{Q} \rightarrow \mathbb{Q}$.

Proof. (1) Since $\left\langle x^{\#}, y\right\rangle=\left\langle y^{b}, x\right\rangle$, the domain of the adjoint of \# contains $\mathcal{Q}$. Hence this domain is dense and \# is closable. A similar argument shows that $b$ is closable. The rest follows from Lemmas 7 and 11 .

(2) Similar to the proof of Lemma 11.

(3) This follows from (1) and Lemma 11. 
(4) If the closable probability algebra $(Q, *, \omega)$ is symmetric, then as in the proof of (1), $\mathbb{Q} \subseteq D\left(S^{*}\right)$. Furthermore, for all $x, y \in \mathbb{Q}$ we have

$$
\left\langle S^{*} x, y\right\rangle=\langle S y, x\rangle=\left\langle y^{*}, x\right\rangle=\left\langle x^{b}, x\right\rangle \text {. }
$$

Hence $S^{*} x=x \in \mathbb{Q}$ and $S^{*}: \mathbb{Q} \rightarrow \mathbb{Q}$. Conversely, if $\mathbb{Q} \subseteq D\left(S^{*}\right)$ and $S^{*}$ : $\mathbb{Q} \rightarrow \mathbb{Q}$, define $x^{b}=S^{*} x$ for any $x \in \mathbb{Q}$. Then for all $x, y \in \mathbb{Q}$ we have

$$
\begin{aligned}
\omega\left(y^{*} x\right) & =\langle x, y\rangle=\left\langle S^{*} y, S x\right\rangle=\left\langle S^{*} y, x^{*}\right\rangle \\
& =\omega\left[x\left(S^{*} y\right)\right]=\omega\left(x y^{b}\right) .
\end{aligned}
$$

Let $(\mathcal{Q}, \#, \omega)$ be a symmetric probability algebra with inner product $\langle x, y\rangle$ $=\omega\left(y^{\sharp} x\right)=\omega\left(x y^{b}\right)$. Let $H$ be the Hilbert space completion of $\mathbb{Q}$. For $x$, $y \in \mathbb{Q}$ define the seminorms $\|x\|_{y}=\|y x\|, \quad y\|x\|=\|x y\|$. The left (right) induced topology on $\mathbb{Q}$ is the topology generated by the collection of seminorms $\left\{\|\|_{y}: y \in \mathbb{Q}\right\}(\{y\|\|: y \in \mathbb{Q}\})$. Let $\mathbb{Q}^{l}\left(\mathbb{Q}^{r}\right)$ be the completion of $\mathbb{Q}$ relative to the left (right) induced topology. Since $\mathbb{Q}$ is dense in $\mathbb{Q}^{l}\left(\mathbb{Q}^{r}\right)$ in the left (right) induced topology, for any $x \in \mathbb{Q}$ there exists a unique operator $\bar{\pi}(x)(\bar{\rho}(x))$ on $\mathbb{Q}^{l}\left(\mathbb{Q}^{r}\right)$ such that $\bar{\pi}(x) y=x y(\bar{\rho}(x) y=y x)$ for all $y \in \mathbb{Q}$. Then $\bar{\pi}$ is a $\#$-representation of $\mathbb{Q}$ with domain $D(\bar{\pi})=\mathbb{Q}^{l} \subseteq H$ and $\bar{\rho}$ is a

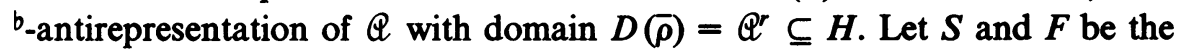
closures of \# and ${ }^{b}$, respectively. Then $Q \subseteq D(S), D(F)$.

LEMMA 17. $D(S)$ is invariant under $S$ and $S^{2} x=x$ for every $x \in D(S)$. $D(F)$ is invariant under $F$ and $F^{2} x=x$ for every $x \in D(F)$. Furthermore $S=F^{*}$ and $F=S^{*}$.

Proof. The first part follows as in the proof of Lemma 12. For the last statement, let $x \in D(S)$ and $y \in D(F)$. There exist a sequence $x_{i} \in \mathbb{Q}$ such that $x_{i} \rightarrow x, S x_{i} \rightarrow S x$ and a sequence $y_{i} \in \mathbb{Q}$ such that $y_{i} \rightarrow y, F y_{i} \rightarrow F y$. We then obtain

$$
\begin{aligned}
\langle S x, y\rangle & =\lim \left\langle S x_{i}, y_{i}\right\rangle=\lim \left\langle x_{i}^{\#}, y_{i}\right\rangle=\lim \left\langle y_{i}^{b}, x_{i}\right\rangle \\
& =\lim \left\langle F y_{i}, x_{i}\right\rangle=\langle F y, x\rangle .
\end{aligned}
$$

It follows that $S=F^{*}$ and $F=S^{*}$.

LeMma 18. Let $A \in \rho(\mathbb{Q})^{\prime}, B \in \pi(\mathbb{Q})^{\prime}$.

(1) $A^{*} 1 \in D\left(\pi^{\sharp}\right)$ if and only if $A y \in D(S)$ for every $y \in \mathbb{Q}$ and in this case $S(A y)=\pi^{\sharp}\left(y^{\sharp}\right) A^{*} 1$.

(2) $B^{*} 1 \in D\left(\rho^{b}\right)$ if and only if $B y \in D(F)$ for every $y \in \mathbb{Q}$ and in this case $F(B y)=\rho^{b}\left(y^{b}\right) B^{*} 1$.

Proof. We shall prove (2), the proof of (1) being similar. Suppose $B^{*} 1 \in$ $D\left(\rho^{b}\right)$. Then for every $x, y \in \mathbb{Q}$ we have

$$
\begin{aligned}
\left\langle x^{\#}, B y\right\rangle & =\left\langle B^{*} \pi\left(x^{\sharp}\right) 1, y\right\rangle=\left\langle B^{*} 1, \pi(x) y\right\rangle \\
& =\left\langle B^{*} 1, \rho(y) x\right\rangle=\left\langle\rho^{b}\left(y^{b}\right) B^{*} 1, x\right\rangle .
\end{aligned}
$$


Hence $B y \in D(F)$ and $F(B y)=\rho^{b}\left(y^{b}\right) B^{*} 1$. Conversely, suppose $B y \in$ $D(F)$ for every $y \in \mathbb{Q}$. As in the proof of Theorem 14, $B^{*} 1 \in D(F)$ and $F\left(B^{*} 1\right)=B 1$. Then for every $y, z \in \mathbb{Q}$ we have

$$
\begin{aligned}
\left\langle\rho(z) y, B^{*} 1\right\rangle & =\left\langle y z, B^{*} 1\right\rangle=\left\langle F\left(B^{*} 1\right), z^{\#} y^{\sharp}\right\rangle=\left\langle B 1, \pi\left(z^{\sharp}\right) y^{\sharp}\right\rangle \\
& =\left\langle B \pi(z) 1, y^{\sharp}\right\rangle=\left\langle B z, y^{\#}\right\rangle=\langle y, F(B z)\rangle .
\end{aligned}
$$

Hence $B^{*} 1 \in D\left(\rho(z)^{*}\right)$ for every $z \in \mathbb{Q}$ and so $B^{*} 1 \in D\left(\rho^{b}\right)$.

Corollary. Let $A \in \rho(\mathbb{Q})^{\prime}, B \in \pi(\mathbb{Q})^{\prime}$. If $\mathbb{Q}^{l}=\mathbb{Q}^{r}$ and $\pi, \rho$ are essentially selfadjoint, then $B y \in D(F), A y \in D(S)$ and $F(B y)=\bar{\rho}\left(y^{b}\right) B^{*} 1, S(A y)=$ $\bar{\pi}\left(y^{\sharp}\right) A^{*} 1$ for every $y \in \mathbb{Q}$.

Proof. If $\mathbb{Q}^{l}=\mathbb{Q}^{r}$ and $\pi, \rho$ are essentially selfadjoint, then $D\left(\pi^{\sharp}\right)=\mathbb{Q}^{l}=$ $\mathbb{Q}^{r}=D\left(\rho^{b}\right)$ and this subspace is invariant under $\rho(\mathbb{Q})^{\prime}$ and $\pi(\mathbb{Q})^{\prime}$.

THEOREM 19. Let $(Q, \#, \omega)$ be a symmetric probability algebra for which $\mathbb{Q}^{l}=\mathbb{Q}^{r}$ and $\pi, \rho$ are essentially selfadjoint. Then $\rho(\mathbb{Q})^{\prime} \subseteq \pi(\mathbb{Q})^{\prime \prime}$.

Proof. Let $A \in \rho(\mathbb{Q})^{\prime}, B \in \pi(\mathbb{Q})^{\prime}$ and let $\mathbb{Q} \ni x_{i} \rightarrow A 1$. Applying the previous corollary, for any $x, y \in \mathbb{Q}$ we have

$$
\begin{aligned}
\langle A B x, y\rangle & =\left\langle B x, A^{*} y\right\rangle=\left\langle S\left(A^{*} y\right), F(B x)\right\rangle \\
& =\left\langle\bar{\pi}\left(y^{\sharp}\right) A 1, \bar{\rho}\left(x^{b}\right) B^{*} 1\right\rangle=\left\langle A 1, \bar{\pi}(y) \bar{\rho}\left(x^{b}\right) B^{*} 1\right\rangle \\
& =\lim \left\langle x_{i}, \bar{\pi}(y) \bar{\rho}\left(x^{b}\right) B^{*} 1\right\rangle=\lim \left\langle y^{\sharp} x_{i} x, B^{*} 1\right\rangle \\
& =\lim \left\langle\pi\left(y^{\sharp}\right) \rho(x) x_{i}, B^{*} 1\right\rangle=\lim \left\langle x_{i}, \bar{\rho}\left(x^{b}\right) \bar{\pi}(y) B^{*} 1\right\rangle \\
& =\left\langle A 1, \bar{\rho}\left(x^{b}\right) \bar{\pi}(y) B^{*} 1\right\rangle=\left\langle\bar{\rho}(x) A 1, \bar{\pi}(y) B^{*} 1\right\rangle \\
& =\left\langle A x, B^{*} y\right\rangle=\langle B A x, y\rangle .
\end{aligned}
$$

Since $A$ and $B$ are bounded we have $A B=B A$ so $\rho(\mathscr{Q})^{\prime} \subseteq \pi(\mathscr{Q})^{\prime \prime}$.

It is natural to conjecture that $\rho(\mathbb{Q})^{\prime}=\pi(\mathbb{Q})^{\prime \prime}$. In the example which follows, this is so even though $\mathbb{Q}^{l} \neq \mathbb{Q}^{r}$.

7. An example. Let $\mathcal{Q}$ be the complex associative algebra with identity 1 generated by elements $p$ and $q$ satisfying the Heisenberg commutation relation $p q-q p=-i 1$. We call $\mathbb{Q}$ the canonical algebra for one degree of freedom. We denote by \# the unique involution of $\mathcal{Q}$ under which the generators $p$ and $q$ are selfadjoint.

The Schrödinger representation $\pi_{0}$ of $(Q, \#)$ acts in the Hilbert space $H_{0}=$ $L^{2}(R)$, with domain the space $S_{0}=\delta(R)$ of infinitely differentiable rapidly decreasing functions, and is defined by $\pi_{0}(p)=p_{0}, \pi_{0}(q)=q_{0}$ where $p_{0}=$ $-i d / d t$ and $q_{0}$ is multiplication by the coordinate $t \in \mathbf{R}$. The Schrödinger representation is faithful, selfadjoint and irreducible, meaning that its commutant $\pi(\mathscr{Q})^{\prime}$ consists of multiples of the identity [13]. The unit vector 


$$
f_{0}(t)=\pi^{-1 / 4} \exp \left(-t^{2} / 2\right)
$$

is strongly cyclic [13].

We denote by $\mathrm{a}^{ \pm}$the linear combinations, mutually adjoint under \#,

$$
a^{+}=2^{-1 / 2}(q-i p), \quad a^{-}=2^{-1 / 2}(q+i p),
$$

satisfying

$$
a^{-} a^{+}-a^{+} a^{-}=1,
$$

and by $a_{0}^{ \pm}$their images under $\pi_{0}$. The vectors

$$
f_{n}=\left\|\left(a_{0}^{+}\right)^{n} f\right\|^{-1}\left(a_{0}^{+}\right)^{n} f_{0}, \quad n=0,1, \ldots,
$$

form a maximal orthonormal set in $H_{0}$ contained in $S_{0}$. In fact $f_{n}$ is the normalized Hermite function

$$
f_{n}(t)=\left(\sqrt{ } \pi 2^{n} n !\right)^{-1 / 2} H_{n}(t) \exp \left(-t^{2} / 2\right)
$$

where

$$
H_{n}(t)=(-1)^{n} \exp t^{2}\left(\frac{d}{d t}\right)^{n} \exp \left(-t^{2}\right)
$$

is the $n$th Hermite polynomial. The action of $a_{0}^{ \pm}$on the $f_{n}$ is

$$
a_{0}^{+} f_{n}=\sqrt{n+1} f_{n+1}, \quad a_{0}^{-} f_{n}= \begin{cases}0 & \text { if } n=0, \\ \sqrt{ } n f_{n-1} & \text { if } n>0 .\end{cases}
$$

We denote by $\mathbb{Q}^{(m)}$ the subspace of $\mathscr{Q}$ comprising polynomials in $p$ and $q$ of degree $<m$. Using (7.3) it is not difficult to see that for $x \in \mathbb{Q}^{(m)}$,

$$
\left\|\pi_{0}(x) f_{n}\right\|<C(x) n^{m / 2}
$$

where $C(x)>0$ is independent of $n$. It follows that for each $\beta>0$ the series

$$
\omega_{\beta}(x)=\left(1-e^{-\beta}\right)^{-1} \sum_{n=0}^{\infty} e^{-n \beta}\left\langle\pi_{0}(x) f_{n}, f_{n}\right\rangle
$$

converges for arbitrary $x \in \mathbb{Q}=\cup_{m=0}^{\infty} \mathbb{Q}^{(m)}$, and defines a faithful state $\omega_{\beta}$ of $(Q, \#)$, called the centered isotropic normal state of parameter $\beta$ (the terminology will be justified in \$9).

LEMMA 19. $\left(Q, \#, \omega_{\beta}\right)$ is a symmetric probability algebra.

Proof. Fix $x \in \mathbb{Q}$ and consider

$$
\begin{aligned}
\omega_{\beta}\left(a^{+} x\right) & =\left(1-e^{-\beta}\right)^{-1} \sum_{n=0}^{\infty} e^{-\beta n}\left\langle a_{0}^{+} \pi_{0}(x) f_{n}, f_{n}\right\rangle \\
& =\left(1-e^{-\beta}\right)^{-1} \sum_{n=0}^{\infty} e^{-\beta n}\left\langle\pi_{0}(x) f_{n}, a_{0}^{-} f_{n}\right\rangle \\
& =\left(1-e^{-\beta}\right)^{-1} \sum_{n=0}^{\infty} e^{-\beta n} \sqrt{n}\left\langle\pi_{0}(x) f_{n}, f_{n-1}\right\rangle
\end{aligned}
$$


together with

$$
\begin{aligned}
\omega_{\beta}\left(x a^{+}\right) & =\left(1-e^{-\beta}\right)^{-1} \sum_{n=0}^{\infty} e^{-\beta n}\left\langle\pi_{0}(x) a_{0}^{+} f_{n}, f_{n}\right\rangle \\
& =\left(1-e^{-\beta}\right)^{-1} \sum_{n=0}^{\infty} e^{-\beta n} \sqrt{n+1}\left\langle\pi_{0}(x) f_{n+1}, f_{n}\right\rangle .
\end{aligned}
$$

Comparing these expressions gives

$$
\omega_{\beta}\left(a^{+} x\right)=e^{-\beta} \omega_{\beta}\left(x a^{+}\right),
$$

and a similar argument gives

$$
\omega_{\beta}\left(a^{-} x\right)=e^{\beta} \omega_{\beta}\left(x a^{-}\right)
$$

Using (7.2), it follows that for arbitrary $x \in \mathcal{Q}$,

$$
\omega_{\beta}(p x)=\omega_{\beta}\left(x p^{\Delta}\right), \quad \omega_{\beta}(q x)=\omega_{\beta}\left(x q^{\Delta}\right)
$$

where

$$
p^{\Delta}=\cosh \beta p-i \sinh \beta q, \quad q^{\Delta}=i \sinh \beta p+\cosh \beta q .
$$

Since $\mathcal{Q}$ is generated by $p$ and $q$ it follows that for arbitrary $x, y \in \mathbb{Q}$,

$$
\omega_{\beta}\left(y^{\sharp} x\right)=\omega_{\beta}\left(x \Delta\left(y^{\sharp}\right)\right)
$$

where $\Delta$ is the automorphism of $\mathscr{Q}$ which maps the generators $p$ and $q$ to $p^{\Delta}$ and $q^{\Delta}$, respectively. Setting $y^{b}=\Delta\left(y^{\sharp}\right)$, we have for all $x, y \in \mathbb{Q}$,

$$
\omega_{\beta}\left(y^{\sharp} x\right)=\omega_{\beta}\left(x y^{b}\right)
$$

as required.

It is clear that the automorphism $\Delta$ is the restriction to $Q$ of the operator denoted also by $\Delta$ whose existence, together with that of the involutory isometric conjugate-linear operator $J$ on the completion $H$ of $\mathcal{Q}$ in the inner product $\langle$,$\rangle determined by \omega$, is asserted in Theorem 15. However, in this case we have much more structure; in fact $(Q, \#, \omega)$ has the properties of a modular Hilbert algebra [17, Definition 1.1], with the exception of the continuity of left multiplication.

THEOREM 20. There exists a complex one-parameter group $\{\Delta(z) ; z \in C\}$ of automorphisms of $\mathbb{Q}$ such that for all $x, y \in \mathbb{Q}, z \in \mathbf{C}, t \in \mathbf{R}$ :

(a) $(\Delta(z) x)^{\#}=\Delta(-\bar{z}) x^{\#}$;

(b) $\langle\Delta(z) x, y\rangle=\langle x, \Delta(\bar{z}) y\rangle$;

(c) $\left\langle\Delta(1) x^{\#}, y^{\sharp}\right\rangle=\langle y, x\rangle$;

(d) $\langle\Delta(z) x, y\rangle$ is an analytic function of $z \in \mathbf{C}$;

(e) $\{x+\Delta(t) x: x \in \mathbb{Q}\}$ is dense in $\mathbb{Q}$.

Proof. We set, for each $z \in \mathbf{C}$, 


$$
\begin{aligned}
& p^{\Delta(z)}=\cosh (\beta z) p-i \sinh (\beta z) q, \\
& q^{\Delta(z)}=i \sinh (\beta z) p+\cosh (\beta z) q .
\end{aligned}
$$

Since $p^{\Delta(z)} q^{\Delta(z)}-q^{\Delta(z)} p^{\Delta(z)}=-i 1$, there is a unique automorphism $\Delta(z)$ of $\mathbb{Q}$ under which $p^{\Delta(z)}$ and $q^{\Delta(z)}$ are the images of $p$ and $q$. By comparing the images of $p$ and $q$ it is clear that $\Delta\left(z_{1}+z_{2}\right)=\Delta\left(z_{1}\right) \Delta\left(z_{2}\right)$ for arbitrary $z_{1}$, $z_{2} \in \mathbf{C}$, and that $\Delta\left(-z_{1}\right)=\Delta\left(z_{1}\right)^{-1}$. Thus $\{\Delta(z): z \in \mathbf{C}\}$ is a complex oneparameter group. Since

$$
p^{\Delta(z) \#}=\cosh (\beta \bar{z}) p+i \sinh (\beta \bar{z}) q=p^{\Delta(-\bar{z})}=p^{\sharp \Delta(-\bar{z})}
$$

and, similarly, $q^{\Delta(z) \#}=q^{\# \Delta(-\bar{z})}$, the conjugate-anti-automorphisms $x \mapsto(\Delta(z) x)^{\#}$ and $x \mapsto \Delta(-z) x^{\#}$ agree on the generators $p$ and $q$ and are, hence, identical. This proves (a). To prove (b) note first that $\Delta(z) a^{+}=e^{-\beta z} a^{+}, \Delta(z) a^{-}=$ $e^{\beta z} a^{-}$, whence $\Delta(z)\left(a^{+j} a^{-k}\right)=e^{-(j-k) \beta z} a^{+j} a^{-k}$ and so

$$
\begin{aligned}
& \left\langle\Delta(z)\left(a^{+j} a^{-k}\right), a^{+l} a^{-m}\right\rangle=e^{-(j-k) \beta z}\left\langle a^{+j} a^{-k}, a^{+l} a^{-m}\right\rangle, \\
& \left\langle a^{+j} a^{-k}, \Delta(\bar{z})\left(a^{+l} a^{-m}\right)\right\rangle=e^{-(l-m) \beta z}\left\langle a^{+j} a^{-k}, a^{+l} a^{-m}\right\rangle .
\end{aligned}
$$

Now if $j-k \neq l-m$ we have

$$
\begin{aligned}
\left\langle a^{+j} a^{-k}, a^{+l} a^{-m}\right\rangle & =\omega_{\beta}\left(a^{+m} a^{-l} a^{+j} a^{-k}\right) \\
& =\left(1-e^{-\beta}\right)^{-1} \sum_{n=0}^{\infty} e^{-\beta n}\left\langle a_{0}^{+m} a_{0}^{-l} a_{0}^{+j} a_{0}^{-k} f_{n}, f_{n}\right\rangle \\
& =\left(1-e^{-\beta}\right)^{-1} \sum_{n=0}^{\infty} e^{-\beta n}\left\langle a_{0}^{+j} a_{0}^{-k} f_{n}, a_{0}^{+l} a_{0}^{-m} f_{n}\right\rangle \\
& =0,
\end{aligned}
$$

as follows from (7.3) and the orthogonality of the $f_{n}$. It follows that for all values of $j, k, l, m$,

$$
\begin{aligned}
\left\langle\Delta(z)\left(a^{+j} a^{-k}\right), a^{+l} a^{-m}\right\rangle & =\delta_{j-k, l-m} e^{-(j-k) \beta}\left\langle a^{+j} a^{-k}, a^{+l} a^{-m}\right\rangle \\
& =\left\langle a^{+j} a^{-k}, \Delta(\bar{z})\left(a^{+l} a^{-m}\right)\right\rangle .
\end{aligned}
$$

Since the elements $a^{+j} a^{-k}, j, k=0,1, \ldots, \operatorname{span} Q,(b)$ is proved. (c) is an immediate consequence of (7.4) and the definition $x^{b}=\Delta x^{\#}=\Delta(1) x^{\sharp}$. (d) is clear from the fact that $\Delta(z) x$ is a polynomial, with coefficients in $\mathcal{Q}$, in the analytic functions $\cosh z, \sinh z$. Finally, since $\Delta(t)\left(a^{+j} a^{-k}\right)=$ $e^{-(j-k) \beta t} a^{+j} a^{-k}$ it is clear that the linear set $\{x+\Delta(t) x: x \in \mathbb{Q}\}$ includes the set $\left\{a^{+j} a^{-k}: j, k=0,1, \ldots\right\}$, linear combinations of which span $\mathbb{Q}$. Hence (e) holds.

The state $\omega_{\beta}$ satisfies the "KMS boundary condition" [6], [17] for the automorphisms $\{\Delta(i t): t \in \mathbf{R}\}$, meaning that for arbitrary $x, y \in \mathbb{Q}$ there is a continuous function $F_{x y}$ on the strip $0<\operatorname{Im} z<1$, analytic in $0<\operatorname{Im} z<1$, such that 


$$
F_{x, y}(t)=\omega_{\beta}((\Delta(i t) x) y), \quad F_{x, y}(t+i)=\omega_{\beta}(y \Delta(i t) x) .
$$

Indeed, if we define $F_{x y}(z)=\omega_{\beta}((\Delta(i z) x) y)$, then $F_{x y}$ is everywhere analytic and, hence, continuous, and, using (a), (b) and (c) of Theorem 20, we have

$$
\begin{aligned}
F_{x y}(t+i) & =\omega_{\beta}((\Delta(i t-1) x) y)=\left\langle y,(\Delta(i t-1) x)^{\sharp}\right\rangle \\
& =\left\langle y, \Delta(i t+1) x^{\sharp}\right\rangle=\left\langle y, \Delta(i t) \Delta(1) x^{\sharp}\right\rangle=\left\langle\Delta(-i t) y, \Delta(1) x^{\sharp}\right\rangle \\
& =\overline{\left\langle\Delta(1) x^{\sharp}, \Delta(-i t) y\right\rangle}=\overline{\left\langle(\Delta(-i t) y)^{\sharp}, x\right\rangle}=\left\langle x, \Delta((-i t) y)^{\sharp}\right\rangle \\
& =\left\langle x, \Delta(-i t) y^{\sharp}\right\rangle=\left\langle\Delta(i t) x, y^{\sharp}\right\rangle=\omega_{\beta}(y \Delta(i t) x)
\end{aligned}
$$

as required.

Motivated by the analogy with a modular Hilbert algebra, we introduce the conjugate-anti-automorphic map

$$
*: \mathbb{Q} \ni x \rightarrow x^{*}=\Delta\left(\frac{1}{2}\right) x^{\#} \text {. }
$$

From (7.5) we obtain $p^{* *}=p, q^{* *}=q$ so ${ }^{*}$ is an involution. Since

$$
\left\langle x^{*}, y^{*}\right\rangle=\left\langle\Delta\left(\frac{1}{2}\right) x^{\#}, \Delta\left(\frac{1}{2}\right) y^{\sharp}\right\rangle=\left\langle\Delta(1) x^{\#}, y^{\sharp}\right\rangle=\langle y, x\rangle,
$$

by Theorem $20(\mathrm{~d}),{ }^{*}$ is isometric and extends uniquely to a conjugate linear isometry $J$ (in fact the operator so denoted of Theorem 15) from $H$ to $H$. In this case we have

Lemma 21. J maps $\mathbb{Q}^{\prime}$ continuously onto $\mathbb{Q}^{r}$, and vice versa. For arbitrary $x \in \mathbb{Q}$,

$$
\bar{\rho}(x)=J \bar{\pi}\left(x^{*}\right) J
$$

Proof. By the isometry of *, for $x, y \in \mathbb{Q}$,

$$
{ }_{y}\left\|x^{*}\right\|=\left\|x^{*} y\right\|=\left\|\left(y^{*} x\right)^{*}\right\|=\left\|y^{*} x\right\|=\|x\|_{y^{*}}
$$

To show that $J: \mathbb{Q}^{l} \rightarrow \mathbb{Q}^{r}$, let $x \in \mathbb{Q}^{l}$ and let $x_{i}$ be a net in $\mathbb{Q}$ converging to $x$ in the left induced topology. Then, in particular, $J x_{i} \rightarrow J x$ in the Hilbert space topology. Moreover, $\rho(y) J x_{i}$ is Cauchy in $H$ for every $y \in \mathbb{Q}$, since

$$
\left\|\rho(y) J x_{i}-\rho(y) J x_{j}\right\|={ }_{y}\left\|x_{i}^{*}-x_{j}^{*}\right\|=\left\|x_{i}-x_{j}\right\|_{y^{*}}
$$

by (7.6). Hence $J x \in D(\overline{\rho(y)})$, where $\overline{\rho(y)}$ is the closure of the operator $\rho(y)$. Since $y$ was arbitrary, $J x \in \bigcap_{y \in \mathbb{Q}} D(\overline{\rho(y)})=D(\bar{\rho})=\mathbb{Q}^{r}$ as required. Extending ${ }_{y}\|\|$ to $\mathbb{Q}^{r}$ and \|\|$_{y}$ to $\mathbb{Q}^{l}$, it follows from (7.5) that

$$
{ }_{y}\|J x\|=\|x\|_{y^{*}}
$$

for all $y \in \mathbb{Q}, x \in \mathbb{Q}^{l}$. Thus $J: \mathbb{Q}^{l} \rightarrow \mathbb{Q}^{r}$ is continuous in the induced topologies. A similar argument shows that $J$ maps $\mathbb{Q}^{r}$ continuously to $\mathbb{Q}^{l}$ and the first statement of the lemma now follows. Finally for arbitrary $y \in \mathbb{Q}^{r}=$ $D(\bar{\rho})$, if $y_{i}$ is a net in $\mathbb{Q}$ converging in $\mathbb{Q}^{r}$ to $y$ we have for arbitrary $x \in \mathbb{Q}$, since $\bar{\pi}\left(x^{*}\right)$ is continuous on $\mathbb{Q}^{l}$, 


$$
\begin{aligned}
J \bar{\pi}\left(x^{*}\right) J y & =\lim _{i} J \bar{\pi}\left(x^{*}\right) J y_{i}=\lim _{i} J \bar{\pi}\left(x^{*}\right) y_{i}^{*}=\lim _{i} J x^{*} y_{i}^{*} \\
& =\lim _{i} J\left(y_{i} x\right)^{*}=\lim _{i} y_{i} x=\bar{\rho}(x) y .
\end{aligned}
$$

8. A commutation theorem. In this section we shall show that, if $\left(Q, \#, \omega_{\beta}\right)$ is the probability algebra generated by the centered isotropic normal state $\omega_{\beta}$ of parameter $\beta$ on the canonical algebra $(Q, \#)$, then the commutants $\pi(\mathcal{Q})^{\prime}$ and $\rho(\mathcal{Q})^{\prime}$ are commutants of each other, and that the isometry $J$ induces a spatial anti-isomorphism from each to the other.

We recall [13] that $\pi(\mathscr{Q})^{\prime}=\bar{\pi}(\mathscr{Q})^{\prime}$ and $\rho(\mathscr{Q})^{\prime}=\bar{\rho}(\mathscr{Q})^{\prime}$. We shall construct a pair of representations $\pi_{1}$ and $\rho_{1}$ unitarily equivalent to the pair $\bar{\pi}$ and $\bar{\rho}$, whose commutants can be exhibited in explicit form.

We recall [14] that the domain $S_{0}=\delta(R)$ of the Schrödinger representation $\pi_{0}$ is a nuclear space with its usual topology, which is in fact the topology on $S_{0}$ induced by $\pi_{0}$ [13], but is determined by the countable family of norms \|\|$_{m}, m=0,1, \ldots$, where

$$
\|f\|_{m}^{2}=\int\left|\left(-\frac{d^{2}}{d t^{2}}+t^{2}+\frac{1}{2}\right)^{m} f(t)\right|^{2} d t=\left\|\pi_{0}\left(\left(a^{-} a^{+}\right)^{m}\right) f\right\|^{2} .
$$

We denote by $H_{0} \otimes \bar{H}_{0}$ the Hilbert space of Hilbert-Schmidt operators in $H_{0}=L^{2}(\mathrm{R})$, and by $S_{0} \otimes \bar{H}_{0}$ the subspace of $H_{0} \otimes \bar{H}_{0}$ consisting of HilbertSchmidt operators whose ranges are contained in $S_{0}$. For $T \in S_{0} \otimes \bar{H}_{0}$ and $x \in \mathcal{Q}, \pi_{0}(x) T$ is clearly a well-defined operator from $H_{0}$ to $S_{0}$.

The authors are grateful to $A$. Katavolos for pointing out an error in an earlier version of the proof of the lemma which follows, and to S. L. Woronowicz for conversations which resulted in the present version.

LEMMA 22. For arbitrary $T \in S_{0} \otimes \bar{H}_{0}$ and $x \in \mathbb{Q}, \pi_{0}(x) T \in S_{0} \otimes \bar{H}_{0}$.

Proof. Since $p_{0}^{2}+q_{0}^{2}$ is an essentially selfadjoint operator with spectrum $\{1,2,3, \ldots\},\left(p_{0}^{2}+q_{0}^{2}\right)^{-1}$ exists and is a Hilbert-Schmidt operator. We write

$$
\pi_{0}(x) T=\left(p_{0}^{2}+q_{0}^{2}\right)^{-1}\left(p_{0}^{2}+q_{0}^{2}\right) \pi_{0}(x) T=\left(p_{0}^{2}+q_{0}^{2}\right)^{-1} \pi_{0}(y) T
$$

where $y=\left(p^{2}+q^{2}\right) x$. Since the Hilbert-Schmidt operators form an ideal in the algebra of all bounded operators, it is sufficient to show that $\pi_{0}(y) T$ is bounded.

To do this we first show that $T$ is closed as an operator from the Hilbert space $H_{0}$ to the topological space $S_{0}$. Indeed, if $g_{n} \rightarrow g$ in $H_{0}$ and $T g_{n} \rightarrow f$ in $S_{0}$ then, in particular, $T g_{n} \rightarrow f$ in the sense of the $H_{0}$-norm on $S_{0}$. Since $T$ is Hilbert-Schmidt it is a closed operator from $H_{0}$ to $H_{0}$ and, hence, $T g=f$.

Since $S_{0}$ is a complete metrizable space it follows from the closed graph theorem that $T$ is continuous from $H_{0}$ to $S_{0}$. Since $\pi_{0}(y)$ is continuous from 
$S_{0}$ to itself, $\pi_{0}(y) T$ is continuous from $H_{0}$ to $S_{0}$. But then $\pi_{0}(y) T$ is, in particular, bounded with respect to the $H_{0}$-norm on $S_{0}$ as required.

It follows from Lemma 22 that, for $x \in \mathbb{Q}, \pi_{1}(x): T \rightarrow \pi_{0}(x) T$ is a well-defined operator in the Hilbert space $H_{0} \otimes \bar{H}_{0}$, with invariant domain $S_{0} \otimes \bar{H}_{0}$.

\section{LEMMA 23. $\pi_{1}$ is a selfadjoint \#-representation of $\mathcal{Q}$.}

Proof. That $\pi_{1}$ is a representation of $\mathcal{Q}$ follows at once from the fact that $\pi_{0}$ is a representation. Also, for arbitrary $T_{1}, T_{2} \in S_{0} \otimes H_{0}$, if $\left\{g_{j}, j=0\right.$, $1, \ldots\}$ is a maximal orthonormal set in $H_{0}$, we have for arbitrary $x \in \mathbb{Q}$,

$$
\begin{aligned}
\left\langle\pi_{1}(x) T_{1}, T_{2}\right\rangle & =\sum_{j=0}^{\infty}\left\langle\left(\pi_{1}(x) T_{1}\right) g_{j}, T_{2} g_{j}\right\rangle \\
= & \sum_{j=0}^{\infty}\left\langle\pi_{0}(x) T_{1} g_{j}, T_{2} g_{j}\right\rangle=\sum_{j=0}^{\infty}\left\langle T_{1} g_{j}, \pi_{0}\left(x^{\sharp}\right) T_{2} g_{j}\right\rangle \\
= & \sum_{j=0}^{\infty}\left\langle T_{1} g_{j},\left(\pi_{1}\left(x^{\sharp}\right) T_{2}\right) g_{j}\right\rangle=\left\langle T_{1}, \pi_{1}\left(x^{\sharp}\right) T_{2}\right\rangle,
\end{aligned}
$$

using the fact that $\pi_{0}$ is a \#-representation.

To prove selfadjointness, let $Q \in D\left(\pi_{1}^{*}\right)$. Then for arbitrary $x \in \mathbb{Q}$ the map $S_{0} \otimes \bar{H}_{0} \ni T \rightarrow\left\langle\pi_{1}(x) T, Q\right\rangle$ is a bounded linear functional in $S_{0} \otimes \bar{H}_{0}$. Set $T=f \otimes \bar{g}$, with $f \in S_{0}, g \in H_{0}$ where, for $g_{1}, g_{2} \in H_{0}, g_{1} \otimes \bar{g}_{2}$ is the element $g \rightarrow\left\langle g, g_{2}\right\rangle g_{1}$ of $H_{0} \cap \bar{H}_{0}$. Then for some positive real number $M$ we have

$$
\begin{aligned}
M\|f\|\|g\| & =M\|f \otimes \bar{g}\| \geqslant\left|\left\langle\pi_{1}(x) f \otimes \bar{g}, Q\right\rangle\right| \\
& =\left|\left\langle\left(\pi_{0}(x) f\right) \otimes \bar{g}, Q\right\rangle\right|=\left|\left\langle\pi_{0}(x) f, Q g\right\rangle\right| .
\end{aligned}
$$

Hence the map $S_{0} \ni f \rightarrow\left\langle\pi_{0}(x) f, Q g\right\rangle$ is a bounded linear functional in $S_{0}$ for all $x \in \mathbb{Q}$. Hence $Q g \in D\left(\pi_{0}^{*}\right)=D\left(\pi_{0}\right)=S_{0}$ since $\pi_{0}$ is selfadjoint. Hence $Q$ maps arbitrary $g \in H_{0}$ into $S_{0}$, that is $Q \in S_{0} \otimes \bar{H}_{0}=D\left(\pi_{1}\right)$. Hence $\pi_{1}$ is selfadjoint.

LEMMA 24. The commutant $\pi_{1}(\mathscr{Q})^{\prime}$ of $\pi_{1}$ is the set of bounded operators $C$ in $H_{0} \otimes \bar{H}_{0}$ of form $C T=T C_{1}$ for some bounded operator $C_{1}$ in $H_{0}$.

Proof. If $T$ is a Hilbert-Schmidt and $C_{1}$ a bounded operator with operator bound $\left\|C_{1}\right\|_{\infty}$, then $T C_{1}$ is a Hilbert-Schmidt operator whose Hilbert-Schmidt norm satisfies $\left\|T C_{1}\right\| \leqslant\|T\|\left\|C_{1}\right\|_{\infty}$ [15]. Hence $C: T \rightarrow T C_{1}$ is a bounded operator in $H_{0} \otimes \bar{H}_{0}$. Moreover, for arbitrary $Q, T \in S_{0} \otimes \bar{H}_{0}$ and $x \in \mathbb{Q}$ we have

$$
\left\langle C \pi_{1}(x) Q, T\right\rangle=\left\langle\pi_{1}(x) Q C_{1}, T\right\rangle=\left\langle Q C_{1}, \pi_{1}(x) T\right\rangle=\left\langle C Q, \pi_{1}\left(x^{\sharp}\right) T\right\rangle,
$$

showing that $C \in \pi(\mathbb{Q})^{\prime}$. 
Conversely, let $C \in \pi(\mathbb{Q})^{\prime}$. For fixed $g, g^{\prime} \in H_{0}$ define a sesquilinear form $\Phi_{g, g^{\prime}}$ on $H_{0} \times H_{0}$ by

$$
\Phi_{g, g^{\prime}}\left(h, h^{\prime}\right)=\left\langle C h \otimes \bar{g}, h^{\prime} \otimes \bar{g}^{\prime}\right\rangle .
$$

Since

$$
\begin{aligned}
\left|\Phi_{g, g^{\prime}}\left(h, h^{\prime}\right)\right| & <\|C\|_{\infty}\|h \otimes \bar{g}\|\left\|h^{\prime} \otimes \bar{g}^{\prime}\right\| \\
& =\|C\|_{\infty}\|h\|\|g\|\left\|h^{\prime}\right\|\left\|g^{\prime}\right\|,
\end{aligned}
$$

$\Phi_{g, g^{\prime}}$ is bounded, hence of form

$$
\Phi_{g, g^{\prime}}\left(h, h^{\prime}\right)=\left\langle\Gamma\left(g, g^{\prime}\right) h, h^{\prime}\right\rangle
$$

for some bounded operator $\Gamma\left(g, g^{\prime}\right)$ with

$$
\left\|\Gamma\left(g, g^{\prime}\right)\right\|_{\infty}<\|C\|_{\infty}\|g\|\left\|g^{\prime}\right\| .
$$

We prove that $\Gamma\left(g, g^{\prime}\right)$ is in the commutant of the Schrödinger representation and is thus a multiple of the identity. Thus, if $f, f^{\prime} \in D\left(\pi_{0}\right)=S_{0}$, for arbitrary $x \in \mathbb{Q}$,

$$
\begin{aligned}
\left\langle\Gamma\left(g, g^{\prime}\right) \pi_{0}\right. & \left.(x) f, f^{\prime}\right\rangle=\Phi_{g, g^{\prime}}\left(\pi_{0}(x) f, f^{\prime}\right) \\
& =\left\langle C\left(\pi_{0}(x) f\right) \otimes \bar{g}, f^{\prime} \otimes \bar{g}^{\prime}\right\rangle=\left\langle C \pi_{1}(x)(f \otimes \bar{g}), f^{\prime} \otimes \bar{g}^{\prime}\right\rangle \\
& =\left\langle C f \otimes \bar{g}, \pi_{1}\left(x^{\sharp}\right) f^{\prime} \otimes \bar{g}^{\prime}\right\rangle=\left\langle C f \otimes \bar{g},\left(\pi_{0}\left(x^{\sharp}\right) f^{\prime}\right) \otimes \bar{g}^{\prime}\right\rangle \\
& =\Phi_{g, g^{\prime}}\left(f, \pi_{0}\left(x^{\sharp}\right) f^{\prime}\right)=\left\langle\Gamma\left(g, g^{\prime}\right) f, \pi_{0}\left(x^{\sharp}\right) f^{\prime}\right\rangle .
\end{aligned}
$$

Hence we can write $\Gamma\left(g, g^{\prime}\right)=\gamma\left(g^{\prime}, g\right) I$ where $\gamma$ is easily seen to be sesquilinear and, in view of (8.1), satisfies

$$
\left|\gamma\left(g^{\prime}, g\right)\right| \leqslant\|C\|_{\infty}\|g\|\left\|g^{\prime}\right\| .
$$

Thus we can set $\gamma\left(g^{\prime}, g\right)=\left\langle C_{1} g^{\prime}, g\right\rangle$ for some bounded operator $C_{1}$ on $H_{0}$. But then, for arbitrary $g, g^{\prime}, h, h^{\prime} \in H_{0}$,

$$
\begin{aligned}
\left\langle C h \otimes \bar{g}, h^{\prime} \otimes \bar{g}^{\prime}\right\rangle=\Phi_{g, g^{\prime}}\left(h, h^{\prime}\right)=\left\langle\Gamma\left(g, g^{\prime}\right) h, h^{\prime}\right\rangle \\
=\gamma\left(g, g^{\prime}\right)\left\langle h, h^{\prime}\right\rangle=\left\langle h, h^{\prime}\right\rangle\left\langle C_{1} g^{\prime}, g\right\rangle=\left\langle h, h^{\prime}\right\rangle\left\langle g^{\prime}, C_{1}^{*} g\right\rangle \\
=\left\langle h \otimes\left(\overline{C_{1}^{*} g}\right), h^{\prime} \otimes \bar{g}^{\prime}\right\rangle=\left\langle(h \otimes \bar{g}) C_{1}, h^{\prime} \otimes \bar{g}^{\prime}\right\rangle .
\end{aligned}
$$

Since elements of form $h \otimes \bar{g}$ are total in $H_{0} \otimes \bar{H}_{0}$, it follows that $C T=T C_{1}$ for arbitrary $T \in H_{0} \otimes \bar{H}_{0}$.

We equip the space $S_{0} \otimes \bar{H}_{0}$ with the topology determined by the countable family of norms \|\|$_{m}, m=0,1, \ldots$, where $\|T\|_{m}=\left\|\pi_{1}\left(\left(a^{-} a^{+}\right)^{m}\right) T\right\|$, and establish an analogue for the topological space $S_{0} \otimes \bar{H}_{0}$ of the " $N$ representation" and the expansion in terms of Hermite functions $f_{n}$ [14] for $S_{0}$. Thus we let $s\left(H_{0}\right)$ be the topological vector space consisting of sequences $\left\{g_{n}\right\}_{n=0}^{\infty}$ of vectors $g_{n} \in H_{0}$ such that, for $m=0,1,2, \ldots, \sup _{n} n^{m}\left\|g_{n}\right\|<$ $\infty$, in which the topology is that determined by the norms \|\|$_{m}, m=0$, $1, \ldots$, where 


$$
\left\|\left\{g_{n}\right\}_{n=0}^{\infty}\right\|_{m}^{2}=\sum_{n}(n+1)^{2 m}\left\|g_{n}\right\|^{2} .
$$

LEMMA 25. The map $S_{0} \otimes \bar{H}_{0} \ni T \mapsto\left\{g_{n}=T^{*} f_{n}\right\}_{n=0}^{\infty}$ is an isomorphism from the topological vector space $S_{0} \otimes \bar{H}_{0}$ onto $s\left(H_{0}\right)$ whose inverse is given by $s_{0}\left(H_{0}\right) \ni\left\{g_{n}\right\}_{n=0}^{\infty} \mapsto T$, where

$$
T=\sum_{n=0}^{\infty} f_{n} \otimes \bar{g}_{n}
$$

the convergence being in the sense of the topology of $S_{0} \otimes \bar{H}_{0}$.

Proof. If $T \in S_{0} \otimes \bar{H}_{0}$ and $g_{n}=T^{*} f_{n}$, then for $m=0,1, \ldots$,

$$
\begin{aligned}
\|T\|_{m}^{2} & =\left\|\pi_{1}\left(\left(a^{-} a^{+}\right)^{m}\right) T\right\|^{2}=\left\|\left(\pi_{1}\left(\left(a^{-} a^{+}\right)^{m}\right) T\right)^{*}\right\|^{2} \\
& =\sum_{n=0}^{\infty}\left\|\left(\pi_{1}\left(\left(a^{-} a^{+}\right)^{m}\right) T\right)^{*} f_{n}\right\|^{2} .
\end{aligned}
$$

Now since $f_{n} \in S_{0}$, for arbitrary $x \in \mathbb{Q}, g \in H_{0}$,

$$
\begin{aligned}
\left\langle\left(\pi_{1}(x) T\right)^{*} f_{n}, g\right\rangle & =\left\langle f_{n}, \pi_{1}(x) T g\right\rangle=\left\langle f_{n}, \pi_{0}(x)(T g)\right\rangle \\
& =\left\langle\pi_{0}\left(x^{\sharp}\right) f_{n}, T g\right\rangle=\left\langle T^{*} \pi_{0}\left(x^{\sharp}\right) f_{n}, g\right\rangle,
\end{aligned}
$$

showing that $\left(\pi_{1}(x) T\right)^{*} f_{n}=T^{*} \pi_{0}\left(x^{\#}\right) f_{n}$. Hence, since $a_{0}^{-} a_{0}^{+} f_{n}=(n+1) f_{n}$ we obtain

$$
\|T\|_{m}^{2}=\sum_{n=0}^{\infty}\left\|T^{*}\left(a_{0}^{-} a_{0}^{+}\right)^{m} f_{n}\right\|^{2}=\sum_{n=0}^{\infty}(n+1)^{m}\left\|T^{*} f_{n}\right\|^{2} .
$$

Hence the map $T \rightarrow\left\{g_{n}=T^{*} f_{n}\right\}_{n=0}^{\infty}$ is isometric for each of the norms \|\|$_{m}$ in $S_{0} \otimes \bar{H}_{0}$ and $s\left(H_{0}\right)$, respectively.

Now let $\left\{g_{n}\right\}_{n=0}^{\infty} \in s\left(H_{0}\right)$, and consider the partial sums $T_{N}=\sum_{n=0}^{N} f_{n} \otimes$ $\bar{g}_{n}, N=0,1, \ldots$, each of which is in $S_{0} \otimes \bar{H}_{0}$ since each $f_{n} \in S_{0}$. For $M>N$, by the orthonormality of the $f_{n}$, we have

$$
\begin{aligned}
\left\|T_{N}-T_{M}\right\|_{m}^{2} & =\left\|\sum_{n=N+1}^{M} f_{n} \otimes \bar{g}_{n}\right\|_{m}^{2}=\left\|\pi_{1}\left(\left(a^{-} a^{+}\right)^{m}\right) \sum_{n=N+1}^{M} f_{n} \otimes \bar{g}_{n}\right\|^{2} \\
& =\left\|\sum_{n=N+1}^{M}\left(\pi_{0}\left(\left(a^{-} a^{+}\right)^{m}\right) f_{n}\right) \otimes \bar{g}_{n}\right\|_{m}^{2} \\
& =\left\|\sum_{n=N+1}^{M}(n+1)^{m} f_{n} \otimes \bar{g}_{n}\right\|^{2} \\
& =\sum_{n=N+1}^{M}(n+1)^{2 m}\left\|f_{n} \otimes \bar{g}_{n}\right\|^{2}=\sum_{n=N+1}^{M}(n+1)^{m}\left\|g_{n}\right\|^{2},
\end{aligned}
$$

which converges to 0 as $M, N \rightarrow \infty$, since $\left\|\left\{g_{n}\right\}_{n=0}^{\infty}\right\|_{m}^{2}$ is finite. Hence 
$\left\{T_{N}\right\}_{N=0}^{\infty}$ is Cauchy in $S_{0} \otimes \bar{H}_{0}$ and so converges to some $T \in S_{0} \otimes \bar{H}_{0}$. Moreover, since $T=\sum_{n=0}^{\infty} f_{n} \otimes \bar{g}_{n}$, in particular in the sense of convergence in Hilbert-Schmidt norm, we have $T^{*}=\sum_{n=0}^{\infty} g_{n} \otimes \bar{f}_{n}$ (Hilbert-Schmidt norm convergence), whence since the $f_{n}$ are orthonormal, $T^{*} f_{n}=g_{n}$. Thus $T$ is indeed the preimage of $\left\{g_{n}\right\}_{n=0}^{\infty}$.

Thus we have an invertible linear map between the two spaces which is isometric for each of the norms defining the respective topologies. Hence the two spaces are isomorphic as claimed.

Since for each $\beta>0$ the sequence $\left\{g_{n}=e^{-n \beta / 2} f_{n}\right\}_{n=0}^{\infty}$ is in $s_{0}\left(H_{0}\right)$, the operator

$$
\Omega_{\beta}=\left(1-e^{-\beta}\right)^{-1 / 2} \sum_{n=0}^{\infty} e^{-n \beta / 2} f_{n} \otimes \bar{f}_{n}
$$

is a well-defined element of $S_{0} \otimes \bar{H}_{0}$.

LEMMA 26. For arbitrary $x \in \mathbb{Q}$,

$$
\omega_{\beta}(x)=\left\langle\pi_{1}(x) \Omega_{\beta}, \Omega_{\beta}\right\rangle \text {. }
$$

Proof.

$$
\begin{aligned}
\left\langle\pi_{1}(x) \Omega_{\beta} \Omega_{\beta}\right\rangle & =\sum_{n=0}^{\infty}\left\langle\left(\pi_{1}(x) \Omega_{\beta}\right) f_{n}, \Omega_{\beta} f_{n}\right\rangle \\
& =\sum_{n=0}^{\infty}\left\langle\pi_{0}(x) \Omega_{\beta} f_{n}, \Omega_{\beta} f_{n}\right\rangle \\
& =\left(1-e^{-\beta}\right)^{-1} \sum_{n=0}^{\infty} e^{-n \beta}\left\langle\pi_{0}(x) f_{n}, f_{n}\right\rangle \\
& =\omega_{\beta}(x) .
\end{aligned}
$$

We recall [13] that a vector $\Omega \in S_{0} \otimes \bar{H}_{0}$ is strongly cyclic for the representation $\pi_{1}$ if for arbitrary $T \in S_{0} \otimes \bar{H}_{0}$ there exists a sequence $x_{n}$ of elements of $Q$ such that $\pi_{1}\left(x_{n}\right) \Omega$ converges to $T$ in the topology determined by the seminorms \|\|$_{y}, y \in \mathbb{Q}$, where $\|T\|_{y}=\left\|\pi_{1}(y) T\right\|$. Since the powers of $a^{-} a^{+}$ dominate $\mathbb{Q}[13]$ this topology coincides with the one already introduced determined by the norms \|\|$_{m}, m=0,1, \ldots$.

LEMMA 27. $\Omega_{\beta}$ is strongly cyclic for $\pi_{1}$.

Proof. We first compute the kernel of the Hilbert-Schmidt operator $\Omega_{\beta}$, considered as an integral operator in $H_{0}=L^{2}(\mathrm{R})$. Using Mehler's formula for Hermite polynomials,

$$
\begin{aligned}
& \sum_{n=0}^{\infty}(n !)^{-1}\left(\frac{1}{2} z\right)^{n} H_{n}(s) H_{n}(t) \\
&=\left(1-z^{2}\right)^{-1 / 2} \exp \left\{\left(1-z^{2}\right)^{-1}\left(2 z s t-z^{2}\left(s^{2}+t^{2}\right)\right)\right\}
\end{aligned}
$$


together with the defining formulae for $\Omega_{\beta}$ and $f_{n}$, this is

$$
\begin{aligned}
F_{\beta}(s, t) & =\left(1-e^{-\beta}\right)^{-1 / 2} \sum_{n=0}^{\infty} e^{-n \beta / 2} f_{n}(s) f_{n}(t) \\
& =\pi^{-1 / 2} \exp \left\{-\frac{1}{2} \operatorname{coth}\left(\frac{1}{2} \beta\right)\left(s^{2}+t^{2}\right)+\operatorname{cosech}\left(\frac{1}{2} \beta\right) s t\right\} .
\end{aligned}
$$

By considering $\left\langle\pi_{1}(x) \Omega_{\beta} g, f\right\rangle=\left\langle\Omega_{\beta} g, \pi_{0}\left(x^{\sharp}\right) f\right\rangle$ for $f \in S_{0}$ it is easily seen that for $x=\Sigma_{m, n} c_{m n} p^{m} q^{n} \in \mathbb{Q}$, the kernel of $\pi_{1}(x) \Omega_{\beta}$ is given by

$$
F_{x}(s, t)=\sum c_{m n}\left(-i \frac{\partial}{\partial s}\right)^{m} s^{n} F_{\beta}(s, t)=P_{x}(s, t) F_{\beta}(s, t)
$$

where $P_{x}$ is a complex polynomial in two indeterminates. Conversely, every such polynomial is of form $P_{x}$ for some $x \in \mathbb{Q}$. To see this, observe that $P_{1}$ is the identity polynomial, that $P_{q x}(s, t)=s P_{x}(s, t)$, and that, since the kernel of $\pi_{1}(p x) \Omega_{\beta}$ is

$$
\begin{aligned}
& \left(-i \frac{\partial}{\partial s}\right) P_{x}(s, t) F_{\beta}(s, t) \\
& \quad=\left(i\left(\operatorname{coth}\left(\frac{1}{2} \beta\right) s-\operatorname{cosech}\left(\frac{1}{2} \beta\right) t\right) P_{x}(s, t)-i \frac{\partial}{\partial s} P_{x}(s, t)\right) F_{\beta}(s, t), \\
& P_{p x}(s, t)=-i \operatorname{cosech}\left(\frac{1}{2} \beta\right) t P_{x}(s, t)+i \operatorname{coth}\left(\frac{1}{2} \beta\right) P_{q x}(s, t)+P^{\prime}(s, t)
\end{aligned}
$$

where $P^{\prime}$ has degree less than that of $P$. It is now clear by induction on the degree of $P$ that an arbitrary polynomial $P$ is of form $P_{x}$ for some $x \in \mathbb{Q}$.

Since the quadratic form in the exponential in $F_{\beta}(s, t)$ is nonsingular, the class of functions $P F_{\beta}$ is dense in the Schwarz space $\delta\left(R^{2}\right)$. Thus, given arbitrary $F \in \delta\left(R^{2}\right)$, there exists a sequence $\left\{x_{N}\right\}_{N=0}^{\infty}$ of elements $x_{N} \in \mathbb{Q}$ such that the kernels $P_{x_{N}} F_{\beta}$ converge to $F$ in the Schwarz topology of $\delta\left(R^{2}\right)$. In particular, $P_{x_{N}} F_{\beta}$ converges to $F$ in each of the norms \|\|$_{m}$ on $\delta\left(R^{2}\right)$, where

$$
\|F\|_{m}^{2}=\int_{R^{2}}\left|\left(-\frac{\partial^{2}}{\partial s^{2}}+t^{2}+\frac{1}{2}\right) F(s, t)\right|^{2} d s d t .
$$

Now each $F \in S\left(R^{2}\right)$ is the kernel of a Hilbert-Schmidt operator $T_{F}$ in $H_{0} \otimes \bar{H}_{0}$. Let us show that $T_{F} \in S_{0} \otimes \bar{H}_{0}$ and that $\|F\|_{m}=\left\|T_{F}\right\|_{m}$. To do this, observe that if

$$
F(s, t)=\sum_{n, n^{\prime}} c_{n, n^{\prime}} f_{n}(s) f_{n^{\prime}}(t)
$$

is the two-dimensional Hermite expansion of $F$ then

$$
\|F\|_{m}^{2}=\sum_{n \cdot n^{\prime}}(n+1)^{2 m}\left|c_{n, n^{\prime}}\right|^{2}
$$


while $T_{F}$ is given by

$$
T_{F}=\sum_{n, n^{\prime}} c_{n, n^{\prime}} f_{n} \otimes \bar{f}_{n^{\prime}}
$$

where the convergence is in Hilbert-Schmidt norm, and this can be expressed as

$$
T_{F}=\sum_{n} f_{n} \otimes \bar{g}_{n}
$$

where $g_{n}=\sum_{n^{\prime}} \bar{c}_{n, n^{\prime}} f_{n^{\prime}}$ and, in consequence,

$$
\begin{aligned}
\left\|\left\{g_{n}\right\}_{n=0}^{\infty}\right\|_{m}^{2} & =\sum_{n}(n+1)^{2 m}\left\|\sum_{n^{\prime}} \bar{c}_{n n^{\prime}} f_{n^{\prime}}\right\|^{2} \\
& =\sum_{n, n^{\prime}}(n+1)^{2 m}\left|c_{n, n^{\prime}}\right|^{2}=\|F\|_{m}^{2}<\infty .
\end{aligned}
$$

It follows from Lemma 25 that $T_{F} \in S_{0} \otimes \bar{H}_{0}$ with

$$
\left\|T_{F}\right\|_{m}^{2}=\left\|\left\{g_{n}\right\}_{n=0}^{\infty}\right\|_{m}^{2}=\|F\|_{m}^{2} .
$$

Now let $T \in S_{0} \otimes \bar{H}_{0}$ be arbitrary; the strong cyclicity of $\Omega_{\beta}$ will be established if we can find elements $x_{N}, N=0,1, \ldots$, of $\mathbb{Q}$ such that for $m=0,1,2, \ldots,\left\|T-\pi_{1}\left(x_{N}\right) \Omega_{\beta}\right\|_{m} \rightarrow 0$ as $N \rightarrow \infty$. We write the "Hermite expansion" of $T$ as

$$
T=\sum_{n=0}^{\infty} f_{n} \otimes \bar{g}_{n}, \quad g_{n}=T^{*} f_{n} \in H_{0}
$$

and recall that this converges in the topology of $S_{0} \otimes \bar{H}_{0}$, so that

$$
\left\|T-\sum_{n=0}^{N} f_{n} \otimes \bar{q}_{n}\right\|_{m} \rightarrow 0
$$

as $n \rightarrow \infty$ for all $m$. Now $S_{0}$ is dense in the Hilbert space $H_{0}$, hence there exist elements $f_{n N} \in S_{0}$ such that $\left\|g_{n}-f_{n N}\right\|<e^{-N}$. We set $T_{N}=\sum_{n=0}^{N} f_{n} \otimes \bar{f}_{n N}$. Then

$$
\begin{aligned}
\left\|T-T_{N}\right\|_{m} & \leqslant\left\|T-\sum_{n=0}^{N} f_{n} \otimes \bar{g}_{n}\right\|_{m}+\left\|\sum_{n=0}^{N} f_{n} \otimes\left(\bar{g}_{n}-f_{n N}\right)\right\|_{m} \\
& =\left\|T-\sum_{n=0}^{N} f_{n} \otimes \bar{g}_{n}\right\|_{m}+\left\{\sum_{n=0}^{N}(n+1)^{2 m} e^{-2 N}\right\}^{1 / 2} .
\end{aligned}
$$

Hence $\left\|T-T_{N}\right\|_{m} \rightarrow 0$ as $N \rightarrow \infty$, for all $m=0,1, \ldots$ Now the kernel of $T_{N}$ is $F_{N}(s, t)=\sum_{n=0}^{N} f_{n}(s) \bar{f}_{n N}(t)$, which is clearly an element of $\delta\left(R^{2}\right)$. It follows that there exist $x_{N M}, M=0,1, \ldots$, such that for all $m=0,1, \ldots$, $\left\|F_{N}-P_{X_{N M}} F_{\beta}\right\|_{m} \rightarrow 0$, or, what is the same thing, $\left\|T_{N}-\pi_{1}\left(x_{N M}\right) \Omega_{\beta}\right\|_{m} \rightarrow 0$, as $M \rightarrow \infty$.

Now choose a sequence $\left\{M_{0}(N)\right\}_{N=0}^{\infty}$ of nonnegative numbers such that, as $N \rightarrow \infty,\left\|T_{N}-\pi_{1}\left(x_{N, M_{0}(N)}\right) \Omega_{\beta}\right\|_{0} \rightarrow 0$. Assuming inductively that a sequence $\left\{M_{m}(N)\right\}_{N=0}^{\infty}$ of nonnegative numbers has been found such that, as $N \rightarrow \infty$, 
$\left\|T_{N}-\pi_{1}\left(x_{N, M_{m}(N)}\right) \Omega_{\beta}\right\|_{m^{\prime}} \rightarrow 0$ for all $m^{\prime}<m$, choose a subsequence $\left\{M_{m+1}(N)\right\}_{N=0}^{\infty}$ of $\left\{M_{m}(N)\right\}_{N=0}^{\infty}$ such that $\left\|T_{N}-\pi_{1}\left(x_{N, M_{m+1}(N)}\right) \Omega_{\beta}\right\|_{m+1} \rightarrow 0$. Finally let $x_{N}$ be defined as $x_{N}=x_{N, M_{N}(N)}$. Then $\left\|T_{N}-\pi_{1}\left(x_{N}\right) \Omega_{\beta}\right\|_{m} \rightarrow 0$ as $N \rightarrow \infty$ for all $m=0,1, \ldots$ Hence by the triangle inequality $\| T-$ $\pi\left(x_{N}\right) \Omega_{\beta} \|_{m} \rightarrow 0, m=0,1, \ldots$, as was to be proved.

Lemmas 26 and 27 show that $\left(H_{0} \otimes \bar{H}_{0}, \pi_{1}, \Omega_{\beta}\right)$ is a Gel'fand-NaimarkSegal triple for the state $\omega_{\beta}$ of $(Q, \#)$ in the sense of [13]. But $(H, \bar{\pi}, 1)$ is trivially a second such triple. By the uniqueness of the Gel'fand-NaimarkSegal construction, there exists a unitary transformation $W_{\beta}$ from $H$ onto $H_{0} \otimes \bar{H}_{0}$ under which $\bar{\pi}$ is unitarily equivalent to $\pi_{1}$, and such that $W_{\beta} \bar{\pi}(x) 1$ $=\pi_{1}(x) \Omega_{\beta}$ for all $x \in \mathcal{Q}$. In particular, it follows from Lemma 24 that $\bar{\pi}$ is selfadjoint. We define the b-antirepresentation $\rho_{1}$ by $\rho_{1}(x)=W_{\beta} \bar{\rho}(x) W_{\beta}^{-1}$ and the conjugate-linear isometry $J_{1}$ from $H_{0} \otimes \bar{H}_{0}$ to itself by $J_{1}=$ $W_{\beta} J W_{\beta}^{-1}$. From Lemma 27 it is clear that $J_{1}$ maps the domain of $\pi_{1}$ bijectively to that of $\rho_{1}$ and vice versa, and that for arbitrary $x \in \mathbb{Q}$, $\rho_{1}(x)=J_{1} \pi_{1}\left(x^{*}\right) J_{1}$.

LEMma 28. For arbitrary $T \in H_{0} \otimes \bar{H}_{0}, J_{1} T=T^{*}$, the operator adjoint of $T$.

Proof. Since $T \mapsto T^{*}$ and $J_{1}$ are both conjugate linear isometries in $H_{0} \otimes$ $\bar{H}_{0}$, we may assume without loss of generality that $T$ belongs to the dense subspace $W_{\beta}(\mathscr{Q})$, say

$$
T=W_{\beta} x=\pi_{1}(x) \Omega_{\beta} .
$$

Then $J_{1} T=W_{\beta} J x=W_{\beta} x^{*}=\pi_{1}\left(x^{*}\right) \Omega_{\beta}$. Thus we must show that $\left(\pi_{1}(x) \Omega_{\beta}\right)^{*}$ $=\pi_{1}\left(x^{*}\right) \Omega_{\beta}$. We may further assume without loss of generality that $x$ is of form $a^{-m} a^{+n}$.

For arbitrary $f \in S_{0}$, since $a_{0}^{+}$is continuous in the topology of $S_{0}$,

$$
\begin{aligned}
a_{0}^{+} \Omega_{\beta} f & =\left(1-e^{-\beta}\right)^{-1 / 2} \sum_{n=0}^{\infty} e^{-n \beta / 2}\left\langle f, f_{n}\right\rangle a_{0}^{+} f_{n} \\
& =\left(1-e^{-\beta}\right)^{-1 / 2} \sum_{N=0}^{\infty} \sqrt{n+1} e^{-n \beta / 2}\left\langle f, f_{n}\right\rangle f_{n+1} \\
& =\left(1-e^{-\beta}\right)^{-1 / 2} e^{\beta / 2} \sum_{n=0}^{\infty} e^{-(n+1) \beta / 2}\left\langle f, a_{0}^{-} f_{n+1}\right\rangle f_{n+1} \\
& =\left(1-e^{-\beta}\right)^{-1 / 2} e^{\beta / 2} \sum_{n^{\prime}=1}^{\infty} e^{-n^{\prime} \beta / 2}\left\langle f, a_{0}^{-} f_{n^{\prime}}\right\rangle f_{n^{\prime}} \\
& =\left(1-e^{-\beta}\right)^{-1 / 2} e^{\beta / 2} \sum_{n^{\prime}=0}^{\infty} e^{-n^{\prime} \beta / 2}\left\langle f, a_{0}^{-} f_{n^{\prime}}\right\rangle f_{n^{\prime}} \\
& =e^{\beta / 2} \Omega_{\beta} a_{0}^{+} f .
\end{aligned}
$$


Since $a_{0}^{+} f \in S_{0}$ we have by repetition

$$
a_{0}^{+n} \Omega_{\beta} f=e^{n \beta / 2} \Omega_{\beta} a_{0}^{+n} f,
$$

and, by a similar argument,

$$
a_{0}^{-m} a_{0}^{+n} \Omega_{\beta} f=e^{-(m-n) \beta / 2} \Omega_{\beta} a_{0}^{-m} a_{0}^{+n} f .
$$

Hence, for arbitrary $g \in H_{0}$,

$$
\begin{aligned}
\left\langle a_{0}^{-m} a_{0}^{+n} \Omega_{\beta} f, g\right\rangle & =e^{-(m-n) \beta / 2}\left\langle\Omega_{\beta} a_{0}^{-m} a_{0}^{+n} f, g\right\rangle \\
& =e^{-(m-n) \beta / 2}\left\langle a_{0}^{-m} a_{0}^{+n} f, \Omega_{\beta} g\right\rangle \\
& =e^{-(m-n) \beta / 2}\left\langle f, a_{0}^{-n} a_{0}^{+m} \Omega_{\beta} g\right\rangle .
\end{aligned}
$$

Since $S_{0}$ is dense in $H_{0}$, it follows that

$$
\left(\pi_{1}(x) \Omega_{\beta}\right)^{*}=\left(a_{0}^{-m} a_{0}^{+n} \Omega_{\beta}\right)^{*}=e^{-\beta(m-n) / 2} a_{0}^{-n} a_{0}^{+m} \Omega_{\beta} .
$$

But it follows easily from (7.5) and the definition of * that

$$
x^{*}=\left(a^{-m} a^{+n}\right)^{*}=e^{-\beta(m-n) / 2} a^{-n} a^{+m} .
$$

Hence $\left(\pi_{1}(x) \Omega_{\beta}\right)^{*}=\pi_{1}\left(x^{*}\right) \Omega_{\beta}$ as required.

Now we can prove the main result of this section.

THEOREM 29. The commutants $\pi(\mathscr{Q})^{\prime}$ and $\rho(\mathfrak{Q})^{\prime}$ of $\pi$ and $\rho$ are commutants of each other as von Neumann algebras. Moreover, the operator $J$ induces a spatial anti-isomorphism from either one of $\pi(\mathbb{Q})^{\prime}, \rho(\mathbb{Q})^{\prime}$ to the other.

Proof. It is sufficient to prove that the commutants of $\pi_{1}$ and $\rho_{1}$ are commutants of each other, spatially isomorphic under $J_{1}$. Since $\rho_{1}(x)=$ $J_{1} \pi_{1}\left(x^{*}\right) J_{1}$, it is clear that $\rho_{1}(\mathbb{Q})^{\prime}=J_{1} \pi_{1}(\mathbb{Q})^{\prime} J_{1}$. By Lemma $24 \rho_{1}(\mathbb{Q})^{\prime}$ thus consists of all operators of form $T \rightarrow\left(T^{*} C_{1}\right)^{*}=C_{1}^{*} T_{1}$ where $C_{1}$ is a bounded operator on $H_{0}$. Thus $\rho_{1}(\mathscr{Q})^{\prime}$ comprises all premultiplications by bounded operators in $H_{0}$, whereas $\pi_{1}(Q)^{\prime}$ consists of all such post-multiplications. But it is well known [4] that these von Neumann algebras are each other's commutants.

9. A uniqueness theorem and its consequences. We recall that, for fixed $\beta>0$, the involution ${ }^{b}$ on the canonical algebra $Q$ defined by $y^{b}=\Delta\left(y^{\sharp}\right)$, where $\Delta$ is the automorphism of $\mathbb{Q}$ under which the images of $p$ and $q$ are $p^{\Delta}=\cosh \beta p-i \sinh \beta q$ and $q^{\Delta}=i \sinh \beta p+\cosh \beta q$, makes $\left(\mathcal{Q}, \#, \omega_{\beta}\right)$ a symmetric probability algebra. The following theorem shows that $\omega_{\beta}$ is determined uniquely by ${ }^{b}$.

THEOREM 30. Let $\omega$ be a state of $(\mathbb{Q}, \#)$ such that for all $x, y \in \mathbb{Q}$, $\omega\left(y^{\sharp} x\right)=\omega\left(x y^{b}\right)$. Then $\omega=\omega_{\beta}$.

Proof. Since $\Delta x=x^{\sharp b}$ we have, for arbitrary $x, y \in \mathbb{Q}$,

$$
\omega(x y)=\omega(y \Delta x) \text {. }
$$


Using the facts that

$$
\Delta a^{+}=e^{-\beta} a^{+}, \quad \Delta a^{-}=e^{\beta} a^{-}
$$

and

$$
a^{-} a^{+}-a^{+} a^{-}=1,
$$

we deduce that, for $r=1,2, \ldots$,

$$
\begin{aligned}
\omega\left(\left(a^{-} a^{+}\right)^{r}\right) & =e^{\beta} \omega\left(\left(a^{+} a^{-}\right)^{r}\right)=e^{\beta} \omega\left(\left(a^{-} a^{+}-1\right)^{r}\right) \\
& =e^{\beta} \sum_{s=0}^{r}\left(\begin{array}{l}
r \\
s
\end{array}\right)(-1)^{s} \omega\left(\left(a^{-} a^{+}\right)^{s}\right) .
\end{aligned}
$$

Equations (9.4) constitute a recurrence relation which determines the quantities $\omega\left(\left(a^{-} a^{+}\right)^{r}\right), r=1,2, \ldots$, once $\omega\left(\left(a^{-} a^{+}\right)^{9}\right)=1$ is fixed. Since our argument is applicable equally well to $\omega_{\beta}$ it follows that $\omega\left(\left(a^{-} a^{+}\right)^{r}\right)=$ $\omega_{\beta}\left(\left(a^{-} a^{+}\right)^{r}\right)$ for all $r=0,1,2, \ldots$ Since $a^{-r} a^{+r}$ can be expressed using (9.3) as a linear combination of $1, a^{-} a^{+},\left(a^{-} a^{+}\right)^{2}, \ldots,\left(a^{-} a^{+}\right)^{r}$ it follows in turn that, for $r=0,1,2 \ldots$,

$$
\omega\left(a^{-r} a^{+r}\right)=\omega_{\beta}\left(a^{-r} a^{+r}\right) .
$$

On the other hand when $r \neq s$ we have, using (9.1) and (9.2),

$$
\omega\left(a^{-r} a^{+s}\right)=e^{r \beta} \omega\left(a^{+s} a^{-r}\right)=e^{(r-s) \beta} \omega\left(a^{-r} a^{+s}\right)
$$

so that $\omega\left(a^{-r} a^{+s}\right)=0$. Since by the same argument $\omega_{\beta}\left(a^{-r} a^{+s}\right)=0$ we deduce that, for $r, s=0,1, \ldots$ and $r \neq s$,

$$
\omega\left(a^{-r} a^{+s}\right)=\omega_{\beta}\left(a^{-r} a^{+s}\right) .
$$

The theorem follows from (9.5) and (9.6) together with the fact that $\mathcal{Q}$ is generated by $a^{+}$and $a^{-}$.

As a first application of Theorem 30 we show that $\omega_{\beta}$ is the unique state satisfying the KMS boundary conditions for the automorphisms $\{\Delta(i t)$ : $t \in R$ \} of Theorem 20. Thus, suppose $\omega$ is a KMS state, so that for arbitrary $x, y \in \mathbb{Q}$ there is a continuous function $F_{x y}$ on the strip $0<\operatorname{Im} z \leqslant 1$, analytic on $0<\operatorname{Im} z<1$ such that

$$
F_{x y}(t)=\omega((\Delta(i t) x) y), \quad F_{x y}(t+i)=\omega(y \Delta(i t) x) .
$$

Define the function $G_{x, y}$ on $\mathbf{C}$ by $G_{x, y}(z)=\omega((\Delta(i z) x) y)$. Then $G_{x, y}$ is everywhere analytic and coincides with $F_{x y}$ on the real axis. Hence $G_{x, y}$ coincides with $F_{x y y}$ everywhere on the domain of definition of the latter, that is, we have

$$
F_{x y}(z)=\omega((\Delta(i z) x) y), \quad 0 \leqslant \operatorname{Im} z<1 .
$$

Now we can write, for arbitrary $x, y \in \mathbb{Q}$, 


$$
\begin{aligned}
\omega\left(y^{\sharp} x\right) & =\omega\left(\left(\Delta(-1) \Delta(1) y^{\sharp}\right) x\right)=F_{\Delta(1) y^{\sharp}, x}(i) \\
& =\omega\left(x \Delta(0) \Delta(1) y^{\sharp}\right)=\omega\left(x y^{b}\right) .
\end{aligned}
$$

It follows from Theorem 30 that $\omega=\omega_{\beta}$.

The involutions \# and ${ }^{b}$ and the modular automorphisms $\Delta(z), z \in \mathbf{C}$, are graded, meaning that they leave invariant each subspace $\mathbb{Q}^{(m)}$ of elements of degree $<m$. The next lemma shows that, modulo a graded automorphism $\nu$ of $\mathbb{Q}$ (which is determined by its action on the generators $p$ and $q$, of form

$$
\left(\begin{array}{l}
p \\
q
\end{array}\right) \rightarrow\left(\begin{array}{l}
p^{\nu} \\
q^{\nu}
\end{array}\right)=A\left(\begin{array}{l}
p \\
q
\end{array}\right)+\left(\begin{array}{ll}
c_{1} & I \\
c_{2} & I
\end{array}\right),
$$

where $A$ is a $2 \times 2$ complex unimodular matrix and $c_{1}, c_{2}$ are complex constants) every graded probability algebra structure on $\mathcal{Q}$ is equivalent to one of the algebras $\left(\mathcal{Q}, \#, \omega_{\beta}\right), 0<\beta<\infty$.

LEMMA 31. Let $\omega$ be a linear form on $\mathcal{Q}$, and suppose that there exist graded involutions ${ }^{\dagger}$ and ${ }^{\natural}$ of $\mathbb{Q}$ such that $\left(\mathbb{Q},{ }^{\dagger}, \omega\right)$ is a symmetric probability algebra for which $\omega\left(y^{\dagger} x\right)=\omega\left(x y^{\natural}\right)$ for all $x$ and $y \in \mathbb{Q}$. Then there exist a positive number $\beta$ and a graded automorphism $\nu$ of $\mathbb{Q}$ such that for all $x \in \mathbb{Q}, \omega(x)=$ $\omega_{\beta}(\nu(x)), x^{\dagger}=\nu^{-1}\left((\nu x)^{\sharp}\right)$ and $x^{\natural}=\nu^{-1}\left((\nu x)^{b}\right)$.

Proof. Let $D$ be the automorphism $x \rightarrow x^{\dagger \natural}$ of $\mathcal{Q}$. Then for arbitrary $x$, $y \in \mathbb{Q}$ we have

$$
\omega(x y)=\omega(y D x),
$$

and $D$ leaves each $\mathbb{Q}^{(m)}$ invariant. Moreover, $D$ is symmetric and positive with respect to the inner product $\langle x, y\rangle=\omega\left(y^{\dagger} x\right)$, being the restriction to $\mathbb{Q}$ of the positive selfadjoint modular operator of Theorem 15 for the closable probability algebra $\left(\mathcal{Q},{ }^{\dagger}, \omega\right)$. Hence the restriction to $\mathcal{Q}^{(1)}$ of $D$ is fully reducible. If it is the identity then setting $x=p$ and $y=q$ in (9.7) gives $\omega(p q)=\omega(q p)$, which is impossible since $\omega(p q)-\omega(q p)=\omega(p q-q p)=-i$. Hence $D$ possesses an eigenvector $a$, say, in $\mathbb{Q}^{(1)}$ for which the corresponding eigenvalue $\lambda \neq 1$. From (9.7) we have $\omega(a x)=\lambda \omega(x a)$ for arbitrary $x \in \mathcal{Q}$, whence, since $\omega$ is ${ }^{\dagger}$-positive, $\omega\left(x a^{\dagger}\right)=\omega\left(a x^{\dagger}\right)=\overline{\lambda \omega\left(x^{\dagger} a\right)}=\bar{\lambda} \omega\left(a^{\dagger} x\right)$. Since $\lambda$ is real $(D$ being symmetric) and nonzero ( $D$ being an automorphism) and since $\omega$ is faithful, it follows that $D a^{\dagger}=\lambda^{-1} a^{\dagger}$. Now the identity 1 is an eigenvector of $D$ in $\mathbb{Q}^{(1)}$ whose eigenvalue is 1 . Thus the elements $1, a, a^{\dagger}$ of $\mathbb{Q}^{(1)}$, being eigenvectors belonging to distinct eigenvalues, are linearly independent. Writing

$$
a=c p+d q+e 1, \quad a^{\dagger}=c^{\prime} p+d^{\prime} q+e^{\prime} 1,
$$

we must therefore have $c d^{\prime}-c^{\prime} d \neq 0$, and hence $a a^{\dagger}-a^{\dagger} a$ is a nonzero multiple of the identity. Since $a a^{\dagger}, a^{\dagger} a$ are ${ }^{\dagger}$-selfadjoint this multiple is real and, by appropriate normalisation of $a$, can be taken to be either +1 or -1 . 
The latter case reduces to the former if we exchange the roles of $a$ and $a^{\dagger}$, hence we may assume that

$$
a a^{\dagger}-a^{\dagger} a=1 .
$$

Since the pair $\left(a, a^{\dagger}\right)$ and $\left(a^{+}, a^{-}\right)$are generators of $Q$ satisfying the same commutation relation there is a graded automorphism $\nu$ of $\mathbb{Q}$ for which $\nu a=a^{-}, \nu a^{\dagger}=a^{+}$. Since $\nu$ maps the mutually ${ }^{\dagger}$-adjoint generators $\left(a, a^{\dagger}\right)$ to

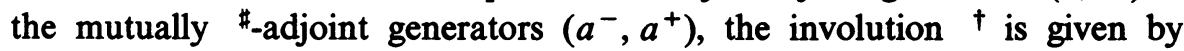
$x^{\dagger}=\nu^{-1}\left((\nu x)^{\#}\right)$.

From (9.8) we have

$$
\omega\left(a a^{\dagger}\right)-\omega\left(a^{\dagger} a\right)=1
$$

while from (9.7)

$$
\omega\left(a a^{\dagger}\right)=\lambda \omega\left(a^{\dagger} a\right) .
$$

From these equations it follows that $\lambda>1$; we write $\lambda=e^{\beta}$ for $\beta$ positive. Then $D a=e^{\beta} a, D a^{\dagger}=e^{-\beta} a^{\dagger}$. Hence $\nu D \nu^{-1} a^{-}=e^{\beta} a^{-}, \nu D \nu^{-1} a^{+}=e^{-\beta} a^{+}$. Comparison with (9.2) shows that the automorphisms $\nu D \nu^{-1}$ and $\Delta$ coincide on the generators $a^{ \pm}$of $\mathcal{Q}$, hence they are identical. Since $x^{\natural}=D\left(x^{\dagger}\right)$ we deduce that the involution ${ }^{\natural}$ is given by

$$
x^{\natural}=\nu^{-1} \Delta \nu x^{\dagger}=\nu^{-1} \Delta(\nu x)^{\sharp}=\nu^{-1}(\nu x)^{b} .
$$

The lemma now follows by applying Theorem 30 to the state $\omega^{\prime}$ of $(\mathbb{Q}, \#)$, where $\omega^{\prime}(x)=\omega\left(\nu^{-1}(x)\right)$, which is \#-positive, since for all $x \in \mathcal{Q}$,

$$
\omega^{\prime}\left(x^{\sharp} x\right)=\omega\left(\nu^{-1}\left[\nu\left(\left(\nu^{-1} x\right)^{\dagger}\right) x\right]\right)=\omega\left(\left(\nu^{-1} x\right)^{\dagger} \nu^{-1} x\right)>0,
$$

and which satisfies

$$
\begin{aligned}
\omega^{\prime}\left(y^{\sharp} x\right) & =\omega\left(\nu^{-1}\left[\nu\left(\left(\nu^{-} y\right)^{\dagger}\right) x\right]\right)=\omega\left(\left(\nu^{-1} y\right)^{\dagger} \nu^{-1} x\right) \\
& =\omega\left(\nu^{-1} x\left(\nu^{-1} y\right)^{\natural}\right)=\omega\left(\nu^{-1}\left[y \nu\left(\left(\nu^{-1} x\right)\right)\right]^{\natural}\right)=\omega^{\prime}\left(x y^{b}\right) .
\end{aligned}
$$

As a final application of Theorem 30 we shall show that the states $\omega_{\beta}$ are, in a certain sense, indeed analogues of normal distribution in classical probability, as suggested by the terminology used to describe them. (Our argument may be compared with that of [2].)

We denote by $\mathbb{Q}_{n}$ the algebraic tensor product $\mathbb{Q} \otimes \cdots \otimes \mathbb{Q}$ of $n$ copies of $\mathcal{Q}$, which we equip with the product involution denoted also by $\#$ for which $\left(x_{1} \otimes \cdots \otimes x_{n}\right)^{\#}=x_{1}^{\#} \otimes \cdots \otimes x_{n}^{\#}$. We set

$$
\begin{aligned}
& p_{j}=1 \otimes \cdots \otimes 1 \otimes \stackrel{(j)}{p} \otimes 1 \otimes \cdots \otimes 1, \\
& q_{j}=1 \otimes \cdots \otimes 1 \otimes \stackrel{(j)}{q} \otimes 1 \otimes \cdots \otimes 1
\end{aligned}
$$


and observe that the elements

$$
p^{(n)}=n^{-1 / 2}\left(p_{1}+\cdots+p_{n}\right), q^{(n)}=n^{-1 / 2}\left(q_{1}+\cdots+q_{n}\right)
$$

are \#-selfadjoint and satisfy the Heisenberg relation $p^{(n)} q^{(n)}-q^{(n)} p^{(n)}=$ $-i$. It follows that there is a \#-isomorphism $\iota_{n}$ from $\mathscr{Q}$ into $\mathbb{Q}^{(n)}$ under which $p$ and $q$ map to $p^{(n)}$ and $q^{(n)}$.

Now let $\omega$ be an arbitrary state of $(\mathscr{Q}, \#)$. The product state $\omega \otimes \cdots \otimes \omega$ on $\left(Q_{n}, \#\right)$ is characterized by the property $\omega \otimes \cdots \otimes \omega\left(x_{1} \otimes \cdots \otimes x_{n}\right)=$ $\omega\left(x_{1}\right) \cdots \omega\left(x_{n}\right)$ where $x_{1}, \ldots, x_{n} \in \mathbb{Q}$, and the formula

$$
\omega^{(n)}(x)=\omega \otimes \cdots \otimes \omega\left(\iota_{n}(x)\right)
$$

defines a new state $\omega^{(n)}$ of $(\mathscr{Q}, \#) . \omega^{(n)}$ is the canonical analogue of the integral resulting from the rescaled $n$-fold convolution $\mu^{(n)}$ of a probability measure $\mu$ on $R$, defined by

$$
\int_{R} f(t) \mu^{(n)}(d t)=\int_{R^{n}} f\left(n^{-1 / 2}\left(t_{1}+\cdots+t_{n}\right)\right) \mu\left(d t_{1}\right) \cdots \mu\left(d t_{n}\right) .
$$

We recall [1] that the distribution determined by $\mu$ is said to be stable if, for each $n=1,2, \ldots$, there exist real numbers $c_{n}$ and $d_{n}$ with $c_{n}>0$ such that

$$
\int f(t) \mu^{(n)}(d t)=\int f\left(c_{n} t+d_{n}\right) \mu(d t)
$$

(this is not the usual definition, but is readily seen to be equivalent to it), that stable distributions are of significance insofar as they are possible limit distributions of sums of identically distributed random variables, and that stable distributions for which the variance is finite are normal. Thus motivated, we shall say that a state $\omega$ of $(\mathcal{Q}, \#)$ is stable if for each $n=1,2, \ldots$ there is a graded automorphism $\nu_{n}$ of $(\mathscr{Q}, \#)$ such that $\omega^{(n)}(x)=\omega\left(\nu_{n}(x)\right)$ for all $x \in \mathbb{Q}$.

We denote by $\omega_{\infty}$ the state of $(\mathscr{Q}, \#)$ given by $\omega_{\infty}(x)=\left\langle\pi_{0}(x) f_{0}, f_{0}\right\rangle$.

Before stating our final theorem we require

LEMMA 32. Let $\omega$ be an arbitrary state of $(\mathcal{Q}, \#)$. Then there exist a graded automorphism $\nu$ of $(\mathcal{Q}, \#)$ and a possibly infinite positive number $\beta$ such that for all elements $x \in \mathbb{Q}^{(2)}$ of degree not exceeding $2, \omega(x)=\omega_{\beta}(\nu(x))$.

Proof. By replacing $\omega$ by $\omega^{\prime}$, where $\omega^{\prime}(x)=\omega\left(\nu_{1}(x)\right)$ and $\nu_{1}$ is the graded automorphism of $(\mathbb{Q}, \#)$ which maps $p$ to $p-\omega(p) 1$ and $q$ to $q-\omega(q) 1$, we may assume that $\omega(p)=\omega(q)=0$. We consider the covariance matrix

$$
\Gamma(\omega)=\left[\begin{array}{cc}
\omega\left(p^{2}\right) & \omega\left(\frac{1}{2}(p q+q p)\right) \\
\omega\left(\frac{1}{2}(p q+q p)\right) & \omega\left(q^{2}\right)
\end{array}\right],
$$

which is the matrix of the quadratic form 


$$
\mathbf{R}^{2} \ni(s, t) \rightarrow \omega\left((s p+t q)^{2}\right) \text {. }
$$

$\Gamma(\omega)$ is thus positive-definite, and by the Heisenberg uncertainty inequality we have also $\operatorname{det} \Gamma(\omega) \geqslant \frac{1}{4}$. It follows that there is a real unimodular $2 \times 2$ matrix $A$ and a real number $\sigma^{2} \geqslant 1$ such that

$$
\Gamma(\omega)=\frac{1}{2} \sigma^{2} A^{t} A \text {. }
$$

Noting that for finite $\beta, \Gamma\left(\omega_{\beta}\right)=\frac{1}{2} \operatorname{coth} \beta I$, while $\Gamma\left(\omega_{\infty}\right)=\frac{1}{2} I$, where $I$ is the $2 \times 2$ identity matrix, it follows that we can write

$$
\Gamma(\omega)=A^{t} \Gamma\left(\omega_{\beta}\right) A
$$

for some $\beta, 0<\beta \leqslant \infty$. But then if $\nu$ is the graded automorphism of $(Q, \#)$ for which the images $p^{\nu}$ and $q^{\nu}$ of $p$ and $q$ are given by

$$
\left(\begin{array}{l}
p^{\nu} \\
q^{\nu}
\end{array}\right)=A\left(\begin{array}{l}
p \\
q
\end{array}\right)
$$

we have $\omega(x)=\omega_{\beta}(\nu(x))$ whenever $x$ is $p^{2}, \frac{1}{2}(p q+q p)$ or $q^{2}$, and hence, since $\omega(x)=\omega_{\beta}(\nu(x))$ for all $x \in \mathbb{Q}^{(1)}$, the lemma is proved.

THEOREM 33. A state $\omega$ of $(\mathscr{Q}, \#)$ is stable if and only if it is of the form $\omega(x)=\omega_{\beta}(\nu(x))$, where $\nu$ is a graded automorphism of $(\mathcal{Q}, \#)$ and $\beta$ is a possibly infinite positive number.

Proof. We prove first that states of the form described are stable. Since it is clear from the definition that if $\omega$ is stable so is $\omega^{\prime}$, where $\omega^{\prime}(x)=\omega(\nu(x))$ for some graded automorphism $\nu$, it suffices to show that $\omega_{\beta}$ is stable for all $\beta$, $0<\beta<\infty$. In fact we shall prove that $\omega_{\beta}^{(n)}=\omega_{\beta}$ for $n=1,2, \ldots$

In the case of finite $\beta$, using the fact that $\iota_{n}$ is both $a$ and a $b_{-}$ isomorphism (where the product involution ${ }^{b}$ on $\mathbb{Q}_{n}$ satisfies $\left(x_{1} \otimes \cdots \otimes\right.$ $\left.x_{n}\right)^{b}=x_{1}^{b} \otimes \cdots \otimes x_{n}^{b}$ for all $\left.x_{1}, \ldots, x_{n} \in \mathbb{Q}\right)$, we have, for arbitrary $x, y \in$ $\mathbb{Q}$, writing

$$
\iota_{n}(x)=\sum_{j} x_{1}^{j} \otimes \cdots \otimes x_{n}^{j}, \quad \iota_{n}(y)=\sum_{k} y_{1}^{k} \otimes \cdots \otimes y_{n}^{k}
$$

with $x_{i}^{j}, y_{l}^{k} \in \mathbb{Q}$,

$$
\begin{aligned}
\omega_{\beta}^{(n)}\left(y^{\sharp} x\right) & =\omega_{\beta} \otimes \cdots \otimes \omega_{\beta}\left(\sum_{j, k} y_{1}^{k \sharp} x_{1}^{j} \otimes \cdots \otimes y_{n}^{k \sharp} x_{n}^{j}\right) \\
& =\sum_{j, k} \omega_{\beta}\left(y_{1}^{k \sharp} x_{1}^{j}\right) \cdots \omega_{\beta}\left(y_{n}^{k \sharp} x_{n}^{j}\right) \\
& =\sum_{j, k} \omega_{\beta}\left(x_{1}^{j} y_{1}^{k b}\right) \cdots \omega_{\beta}\left(x_{n}^{j} y_{n}^{k b}\right) \\
& =\omega_{\beta} \otimes \cdots \otimes \omega_{\beta}\left(\sum_{j, k} x_{1}^{j} y_{1}^{k b} \otimes \cdots \otimes x_{n}^{j} y_{n}^{k b}\right) \\
& =\omega_{\beta}^{(n)}\left(x y^{b}\right) .
\end{aligned}
$$


It follows from Theorem 30 that $\omega_{\beta}^{(n)}=\omega_{\beta}$ as asserted.

In the case $\beta=\infty$ we note that $\omega_{\infty}$ is characterized by the fact that

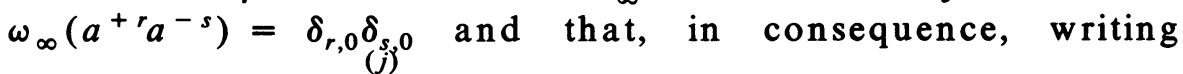
$a_{j}^{ \pm}=1 \otimes \cdots \otimes 1 \otimes a^{ \pm} \otimes 1 \otimes \cdots \otimes 1$,

$$
\begin{aligned}
& \omega_{\infty}^{(n)}\left(a^{+r} a^{-s}\right) \\
& \quad=\omega_{\infty} \otimes \cdots \otimes \omega_{\infty}\left(n^{-(r+s) / 2}\left(a_{1}^{+}+\cdots+a_{n}^{+}\right)^{r}\left(a_{1}^{-}+\cdots+a_{n}^{-}\right)^{s}\right) \\
& \quad=\delta_{r, 0} \delta_{s, 0},
\end{aligned}
$$

as may be seen from the multinomial expansion of $\left(a_{1}^{+}+\cdots+a_{n}^{+}\right)^{r}$ and $\left(a_{1}^{-}+\cdots+a_{n}^{-}\right)^{s}$. Hence $\omega_{\infty}^{(n)}=\omega_{\infty}$.

Conversely let $\omega$ be a stable state of $(\mathcal{Q}, \#)$. By Lemma 32 there exist $\beta$, $0<\beta<\infty$, and a graded \#-automorphism $\nu$ of $\mathscr{Q}$ such that $\omega(x)=\omega_{\beta}(\nu(x))$ for all $x \in \mathbb{Q}^{(2)}$. Since if $\omega$ is stable, so too is $\omega^{\prime}$, where $\omega^{\prime}(x)=\omega\left(\nu^{-1}(x)\right)$, we may assume that in fact $\omega$ coincides with $\omega_{\beta}$ on $\mathbb{Q}^{(2)}$. We shall prove that under this assumption $\omega=\omega_{\beta}$.

Suppose first that $\beta=\infty$. Then $\omega\left(a^{+} a^{-}\right)=\omega_{\infty}\left(a^{+} a^{-}\right)=0$. It follows from Schwarz's inequality that $\omega$ vanishes on the left ideal in $Q$ generated by $a^{-}$and on the right ideal generated by $a^{+}$. Hence $\omega\left(a^{+r} a^{-s}\right)=\delta_{r, 0} \delta_{s, 0}=$ $\omega_{\infty}\left(a^{+r} a^{-s}\right)$ for all $r, s=0,1,2 \ldots$ Hence $\omega=\omega_{\infty}$.

Now let $\beta$ be finite. For arbitrary $x, y \in \mathbb{Q}^{(1)}$,

$$
\omega\left(y^{\sharp} x\right)=\omega_{\beta}\left(y^{\sharp} x\right)=\omega_{\beta}\left(x y^{b}\right)=\omega\left(x y^{b}\right) .
$$

We shall prove that $\omega\left(y^{\sharp}\right)=\omega\left(x y^{b}\right)$ for arbitrary $x, y \in \mathbb{Q}$; from this it will follow from Theorem 30 that $\omega=\omega_{\beta}$. It is sufficient to consider the case when $y=r$ and $x$ is of form $r^{1} \ldots r^{N}$ where each of $r, r^{1}, \ldots, r^{N}$ is either $p$ or $q$. The proof is by induction on $N$, the case $N=1$ being already established.

Since $\omega$ is stable, for $n=1,2, \ldots$ there is a graded automorphism $\nu_{n}$ of $(\mathbb{Q}, \#)$ such that $\omega^{(n)}(z)=\omega\left(\nu_{n}(z)\right)$ for arbitrary $z \in \mathbb{Q}$. Since $\omega^{(n)}(p)=$ $n^{1 / 2} \omega(p)=0$ and $\omega^{(n)}(q)=n^{-1 / 2} \omega(q)=0$, the automorphism $\nu_{n}$ acts on $p$ and $q$ by

$$
\left(\begin{array}{l}
p^{\nu_{n}} \\
q^{\nu_{n}}
\end{array}\right)=A_{\dot{n}}\left(\begin{array}{l}
p \\
q
\end{array}\right)
$$

where $A_{n}$ is a real unimodular $2 \times 2$ matrix, and the covariance matrices $\Gamma(\omega)$ and $\Gamma\left(\omega^{(n)}\right)$ of $\omega$ and $\omega^{(n)}$ are related by

$$
\Gamma\left(\omega^{(n)}\right)=A_{n}^{t} \Gamma(\omega) A_{n} .
$$

But it is easily verified from the definition of $\omega^{(n)}$, using the vanishing of $\omega(p)$ and $\omega(q)$, that $\Gamma\left(\omega^{(n)}\right)$ and $\Gamma(\omega)$ are in fact equal. Since $\Gamma(\omega)=\Gamma\left(\omega_{\beta}\right)$ and the latter is a multiple of the identity, it follows from (9.9) that $A_{n}$ is an orthogonal matrix and hence, being proper, is a rotation matrix. Hence we 
can write

$$
p^{\nu_{n}}=\cos \theta_{n} p+\sin \theta_{n} q, \quad q^{\nu_{n}}=-\sin \theta_{n} p+\cos \theta_{n} q
$$

for some real $\theta_{n}$, and also

$$
p^{\nu_{n}^{-1}}=\cos \theta_{n} p-\sin \theta_{n} q, \quad q^{\nu_{n}^{-1}}=\sin \theta_{n} p+\cos \theta_{n} q
$$

Now consider

$$
\begin{aligned}
\omega\left(y^{\sharp} x\right)-\omega\left(x y^{b}\right) & =\omega\left(y^{\sharp} x-x y^{b}\right)=\omega^{(n)}\left(\nu_{n}^{-1}\left(y^{\sharp} x-x y^{b}\right)\right) \\
& =\omega^{(n)}\left(y^{\prime \sharp} x^{\prime}-x^{\prime} y^{\prime b}\right)
\end{aligned}
$$

where, for $z \in \mathbb{Q}, z^{\prime}=\nu_{n}^{-1} z$. Writing $r_{j}^{\prime}=1 \otimes \cdots \otimes 1 \otimes \stackrel{(j)}{r^{\prime} \otimes 1 \otimes \cdots \otimes}$ $1, r_{j}^{(k) \prime}=1 \otimes \cdots \otimes 1 \otimes \stackrel{(j)}{r}^{(k)^{\prime}} \otimes 1 \otimes \cdots \otimes 1$, and using the definition of $\omega^{(n)}$ this becomes $n^{-(N+1) / 2} \omega \otimes \cdots$

$$
\otimes \stackrel{(n)}{\omega}\left[\left(\sum_{j=1}^{n} r_{j}^{\prime}\right)^{\#} \prod_{k=1}^{N}\left(\sum_{j=1}^{n} r_{j}^{(k) \prime}\right)-\prod_{k=1}^{N}\left(\sum_{j=1}^{n} r_{j}^{(k) \prime}\right)\left(\sum_{j=1}^{n} r_{j}^{\prime}\right)^{b}\right] .
$$

Expressing the two products of sums as sums of products we observe that corresponding products which involve more than one value of the suffix $j$ cancel by the inductive hypothesis. The terms remaining are

$$
\begin{aligned}
n^{-(N+1) / 2} \sum_{j=1}^{n} & \omega \otimes \cdots \otimes \otimes_{\omega}^{(n)}\left(r_{j}^{\prime \#} \prod_{k=1}^{N} r_{j}^{(k) \prime}-\prod_{k=1}^{N}\left(r_{j}^{(k) \prime}\right) r_{j}^{\prime b}\right) \\
& =n^{-(N-1) / 2} \omega\left(r^{\prime \#} \prod_{k=1}^{N} r^{\prime(k)}-\prod_{k=1}^{N} r^{(k)} r^{\prime b}\right) \\
& =n^{-(N-1) / 2} \omega\left(\nu_{n}^{-1}\left(y^{\sharp} x-x y^{b}\right)\right) .
\end{aligned}
$$

Now from (9.10) it is clear that $\omega\left(\nu_{n}^{-1}\left(y^{\sharp} x-x y^{b}\right)\right)$ is a polynomial in $\cos \theta_{n}$ and $\sin \theta_{n}$ whose coefficients do not depend on $n$. Hence $\omega\left(\nu_{n}^{-1}\left(y^{\sharp} x-x y^{b}\right)\right)$ remains bounded as $n \rightarrow \infty$. Taking this limit we deduce that $\omega\left(y^{\sharp} x\right)-$ $\omega\left(x y^{b}\right)=0$ as required.

\section{REFERENCES}

1. L. Breiman, Probability, Addison-Wesley, Reading, Mass., 1968.

2. C. D. Cushen and R. L. Hudson, A quantum-mechanical central limit theorem, J. Appl. Probability 8 (1971), 454-469.

3. A. van Daele, $A$ new approach to the Tomita-Takesaki theory of generalized Hilbert algebras, J. Functional Analysis 15 (1974), 378-393. 
4. J. Dixmier, Les algèbres d'opérateurs dans l'espace Hilbertien (Algèbres de von Neumann), 2nd ed., Gauthier-Villars, Paris, 1969.

5. H. A. Dye, The Radon-Nikodym theorem for finite rings of operators, Trans. Amer. Math. Soc. 72 (1952), 243-280.

6. G. G. Emch, Algebraic methods in statistical mechanics and quantum field theory, Wiley, New York, 1972.

7. I. M. Gel'fand and N. Ya. Vilenkin, Generalized functions, Vol. 4, Academic Press, New York, 1964.

8. S. P. Gudder and J.-P. Marchand, Probability theory on von Neumann algebras, J. Mathematical Phys. 13 (1972), 799-806.

9. S. P. Gudder and D. Strawther, Orthogonally additive and orthogonally monotone functions on vector spaces, Pacific J. Math. 58 (1975), 427-436.

10. P. R. Halmos and L. J. Savage, Application of the Radon-Nikodym theorem to the theory of sufficient statistics, Ann. Math. Statist. 20 (1949), 225-241.

11. M. A. Naimark, Normed algebras, 3rd ed., Wolters-Noordhoff, Gröningen, 1972.

12. M. Nakumara, M. Takesaki and H. Umegaki, $A$ remark on the expectations of operator algebras, Kōdai Math. Sem. Rep. 12 (1960), 82-90.

13. R. T. Powers, Self-adjoint algebras of unbounded operators, Comm. Math. Phys. 21 (1971), 85-124.

14. M. Reed and B. Simon, Methods of mathematical physics. Vol. 1: Functional analysis, Academic Press, New York, 1972.

15. R. Schatten, Norm ideals of completely continuous operators, Springer, Berlin, 1970.

16. I. E. Segal, Non-commutative extension of abstract integration, Ann. of Math. (2) 57 (1953), $401-457$.

17. M. Takesaki, Tomita's theory of modular Hilbert algebras and its applications, Lecture Notes in Math., vol. 128, Springer-Verlag, Berlin, 1970.

18. H. Umegaki, Conditional expectation in an operator algebra. II, Tôhoku Math. J. 8 (1956), 86-100.

Department of Mathematics, University of Denver, University Park, Denver, COLORADO 80210

Department of Mathematics, University of Nottingham, University Park, Nottingham NG7 2RD, ENGLAND 